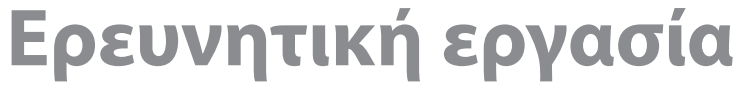 Research article
}

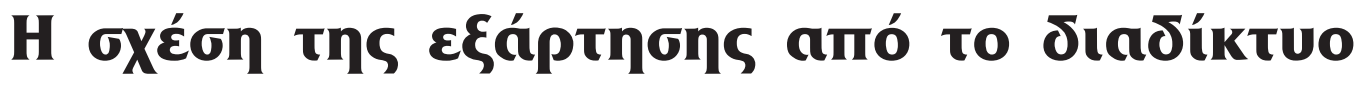

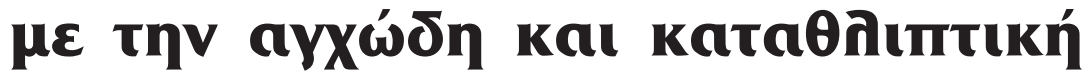

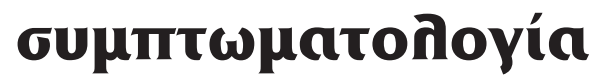

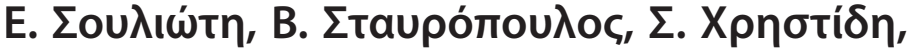

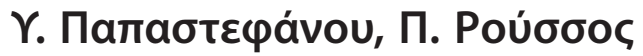

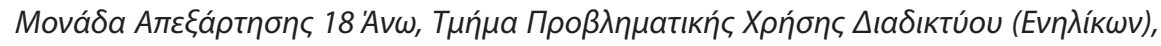

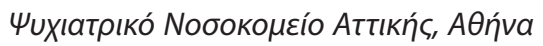 \\ Uuxıatpıкń 2018, 29:160-171
}

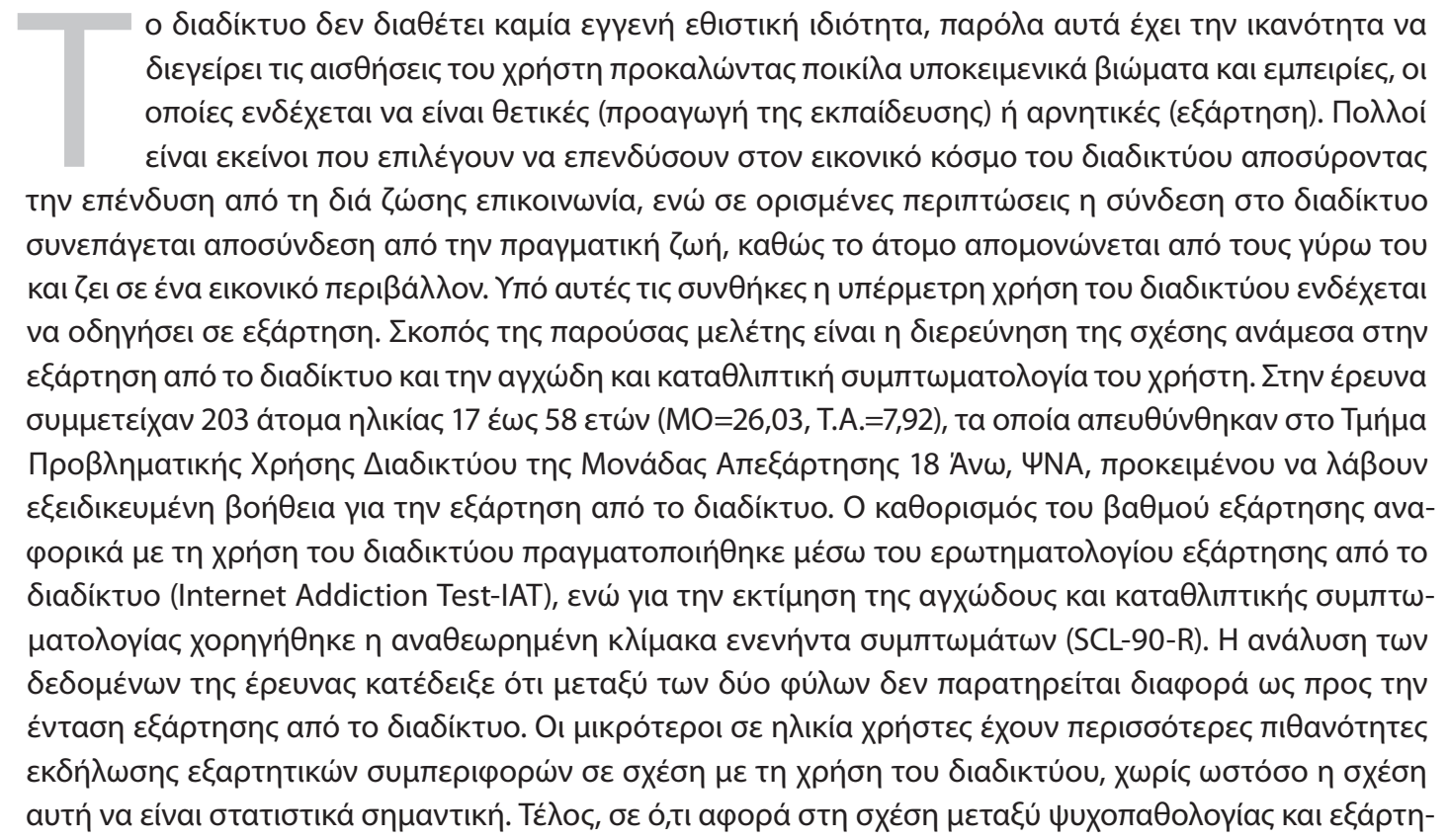




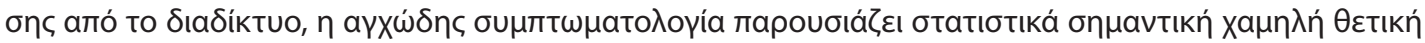

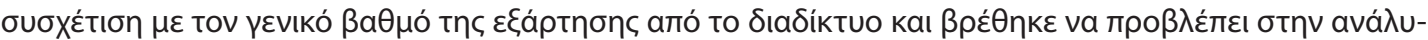

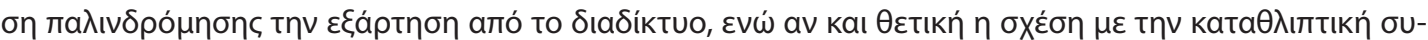

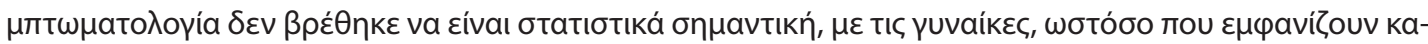

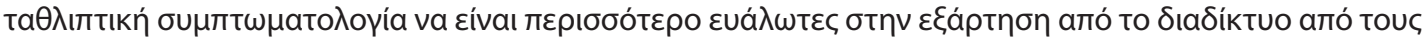

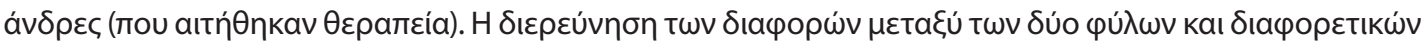

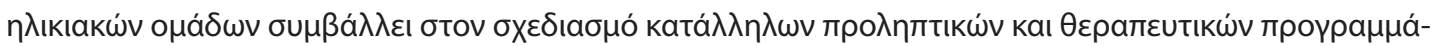

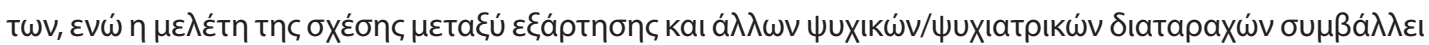

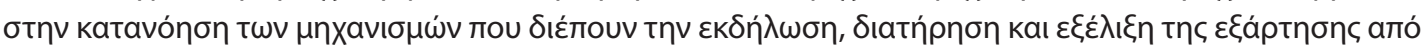

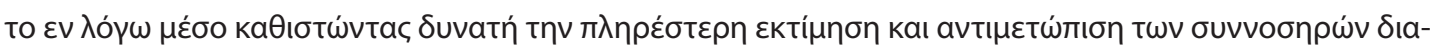

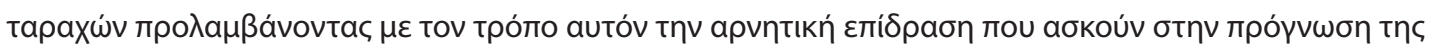

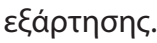

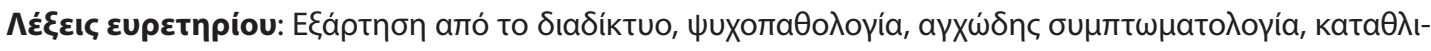

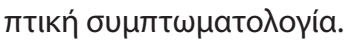

\section{Eıăywyń}

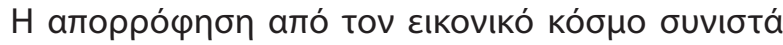

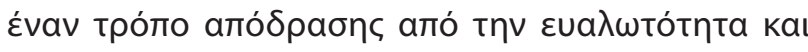

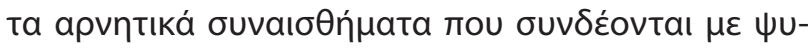

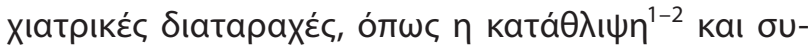

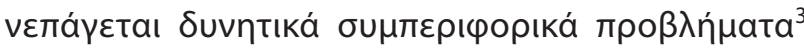

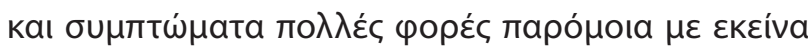

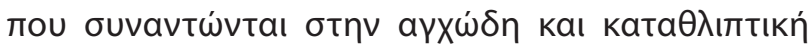

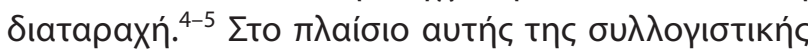

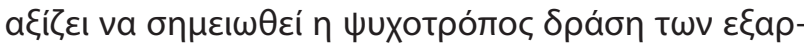

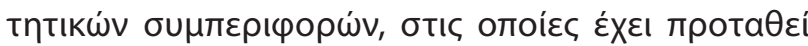

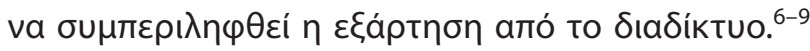

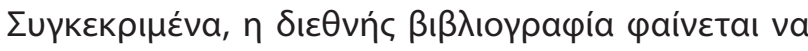

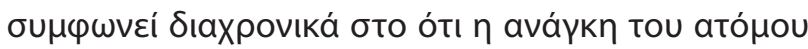

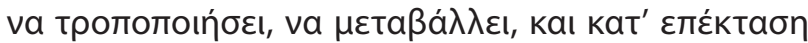

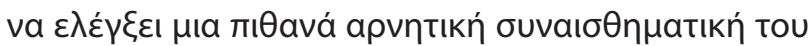

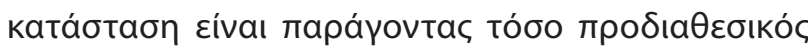

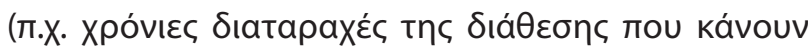

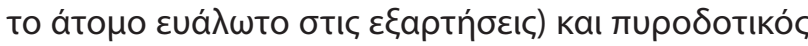

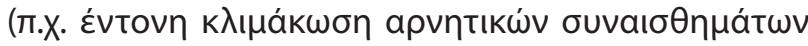

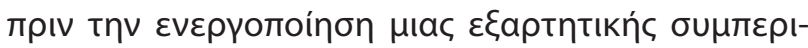

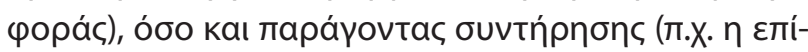

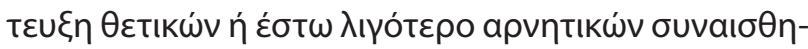

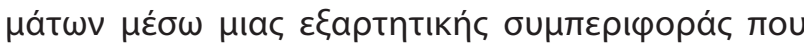

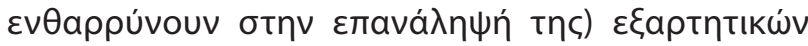

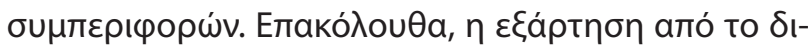

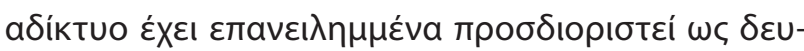

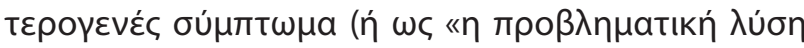

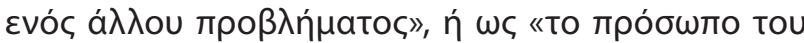

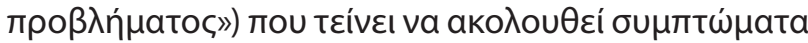

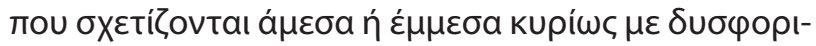

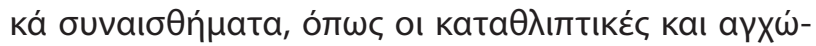

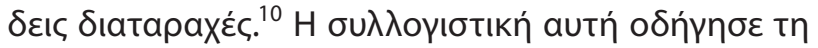

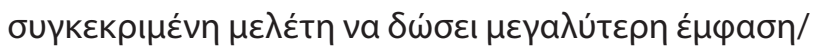

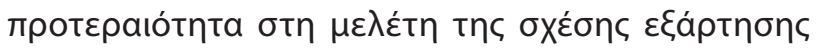

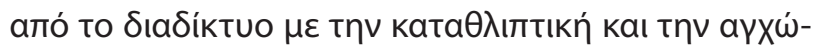

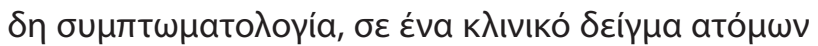

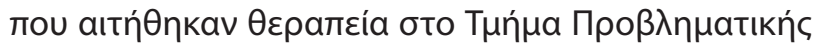

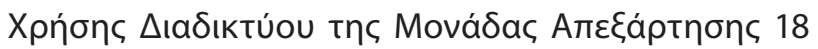

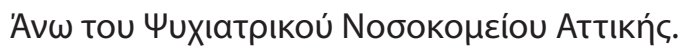

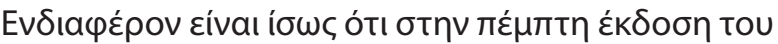

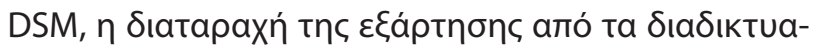

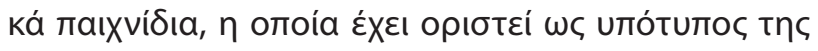

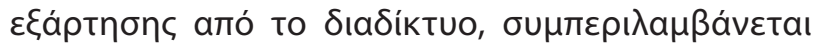

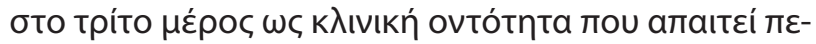

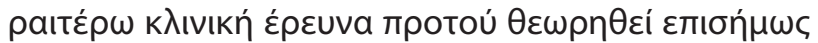

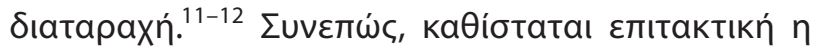

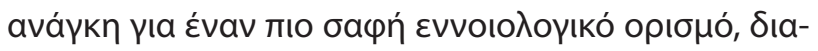

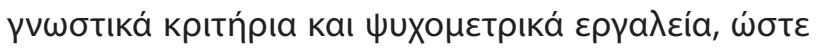

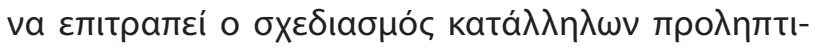

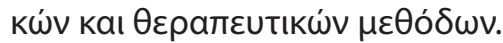

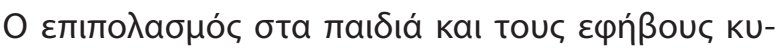

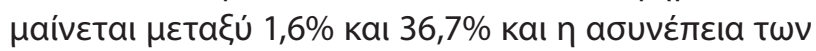

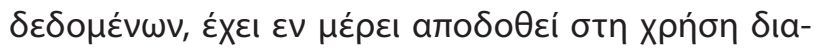

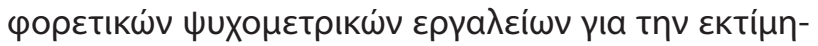

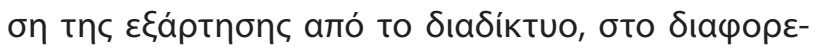

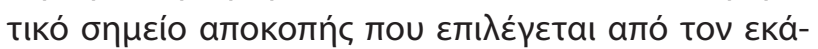

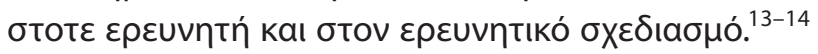

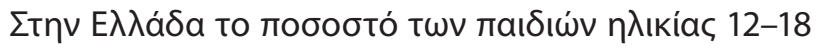

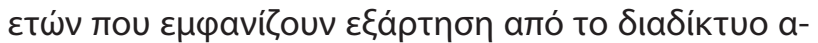

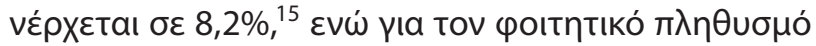




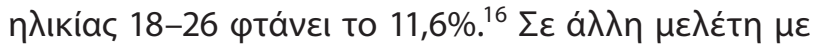

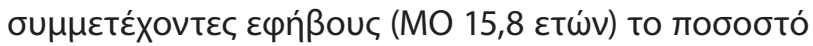

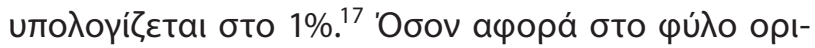

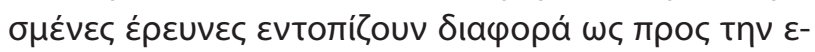

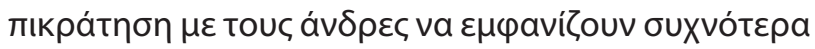

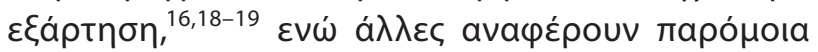

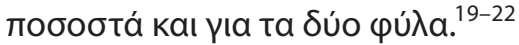

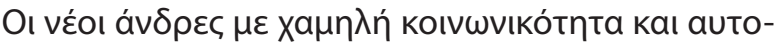

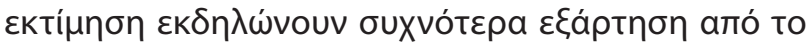

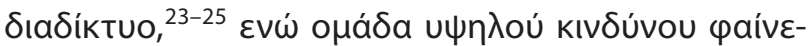

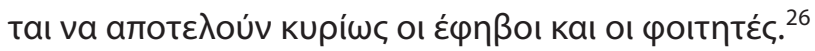

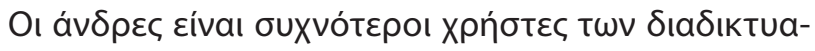

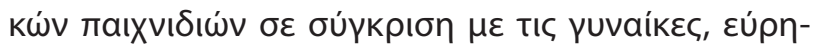

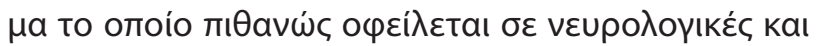

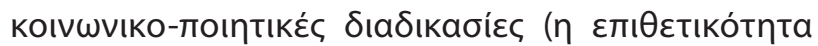

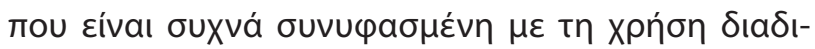

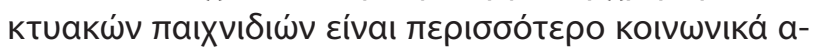

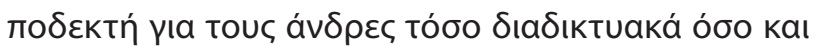

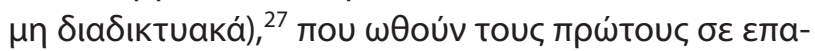

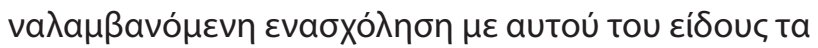

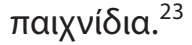

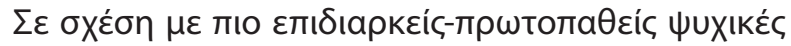

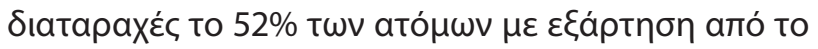

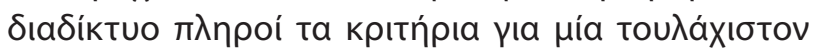

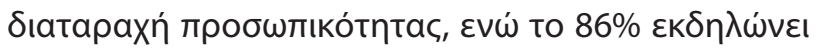

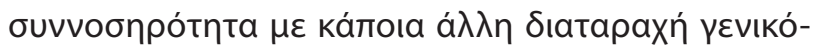

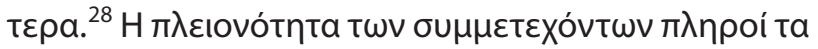

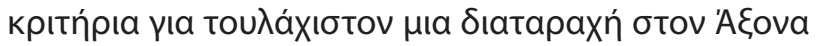

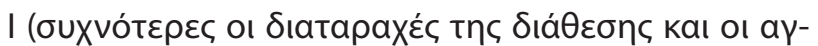

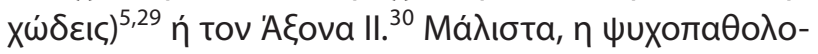

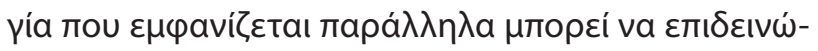

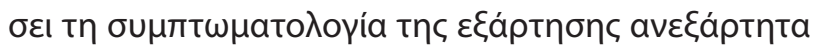

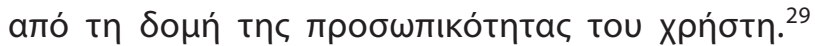

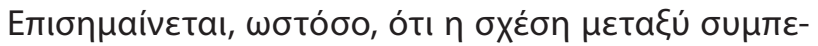

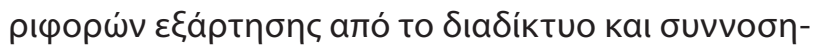

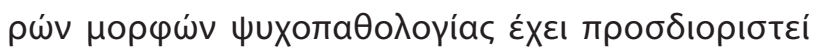

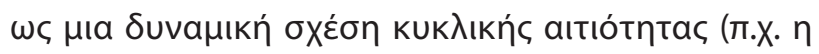

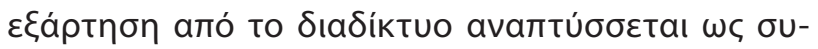

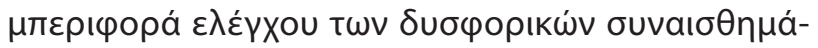

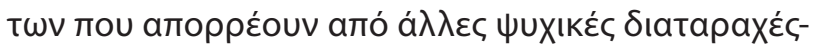

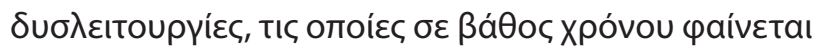
va $\varepsilon \pi ı \delta \varepsilon ı v \omega ́ v \varepsilon ı .{ }^{6}$

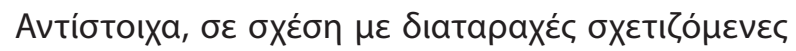

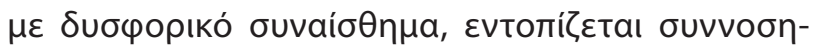

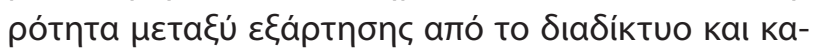

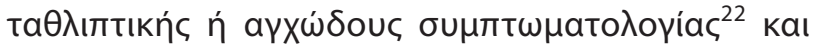

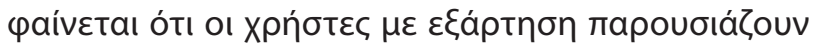

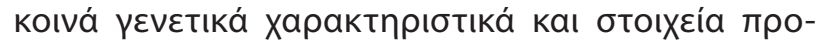

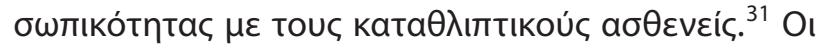

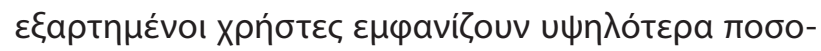

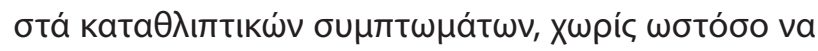

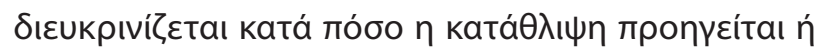

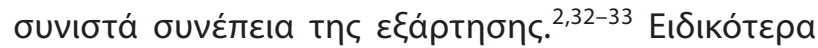

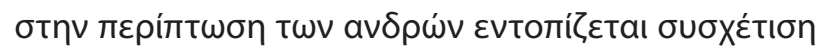

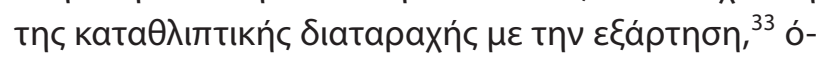

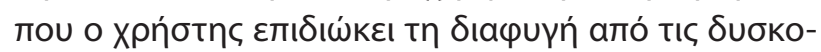

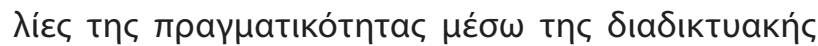

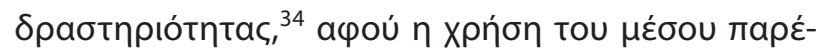

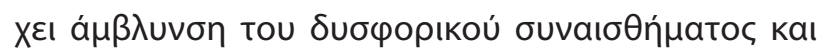

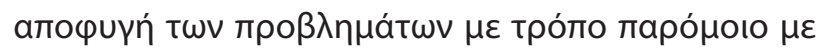

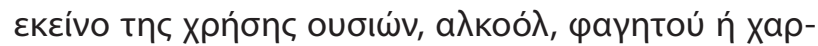

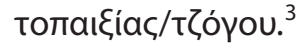

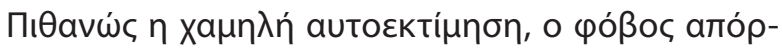

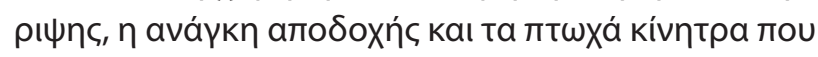

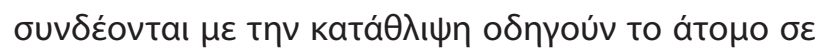

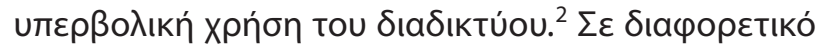

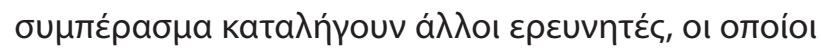

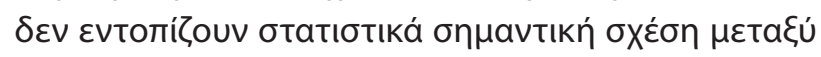

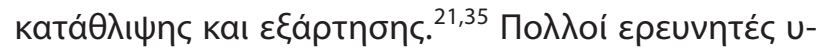

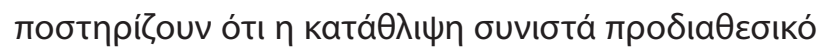

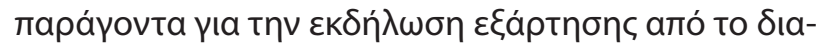

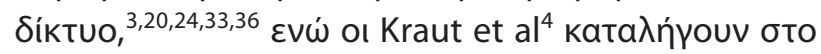

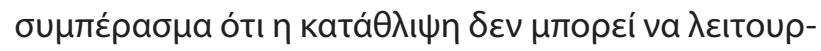

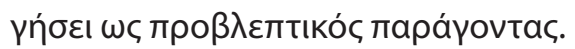

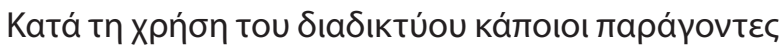

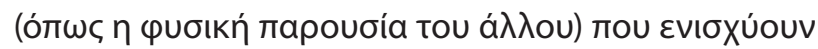

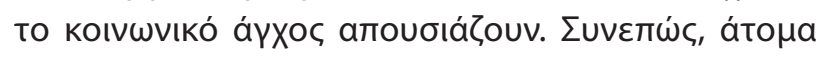

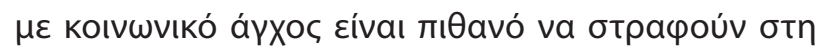

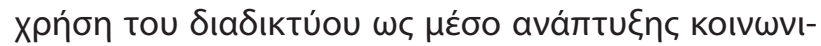

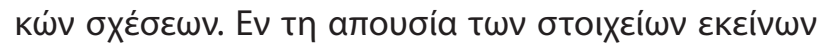

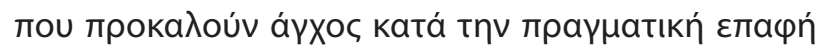

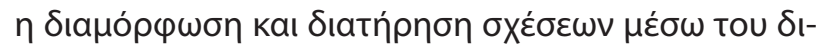

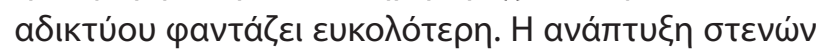

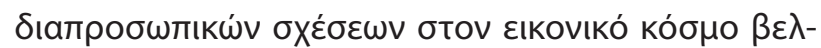

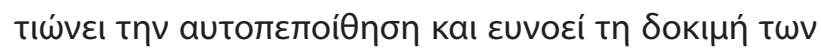

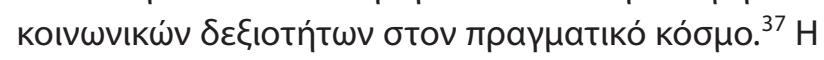

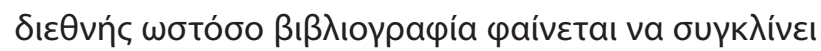

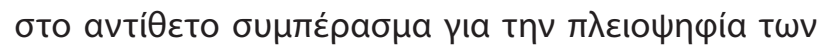

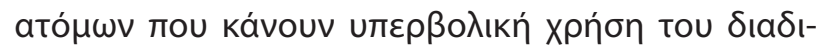

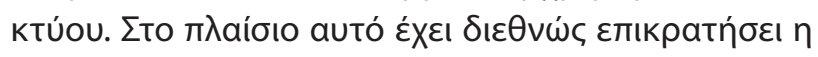

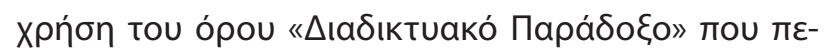

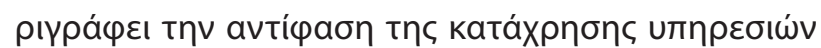

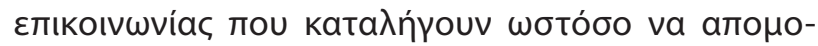




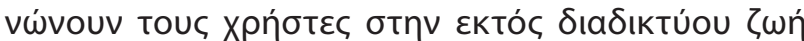
Touc. ${ }^{38}$

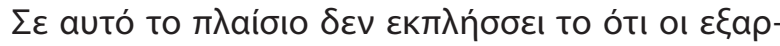

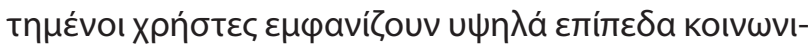

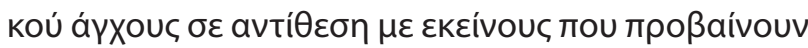

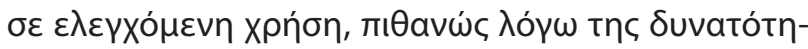

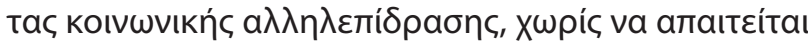

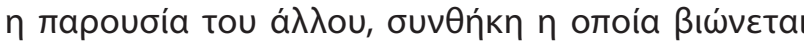

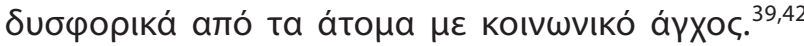

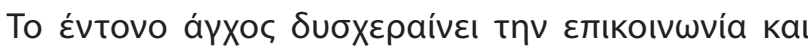

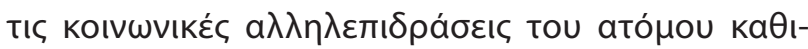

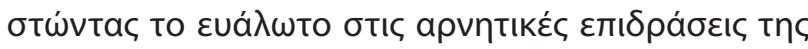

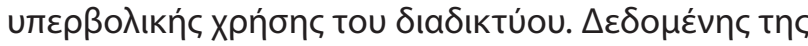

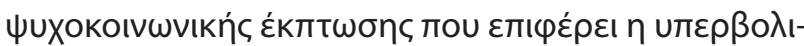

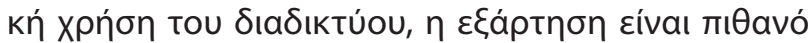
va $\varepsilon \pi$

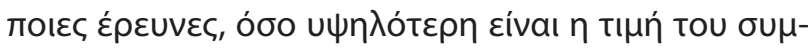

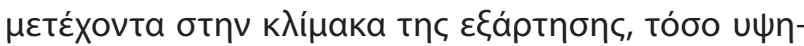

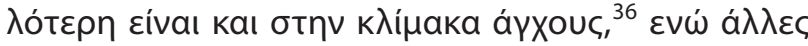

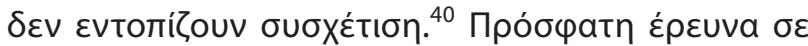

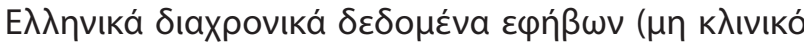

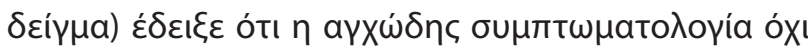

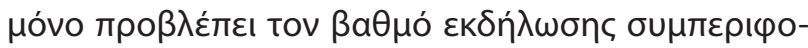

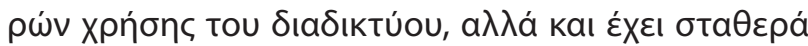

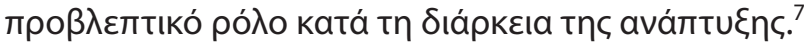

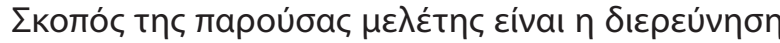

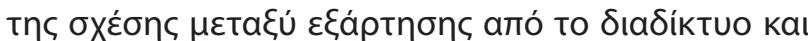

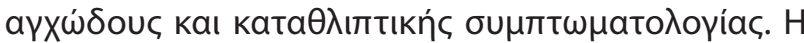

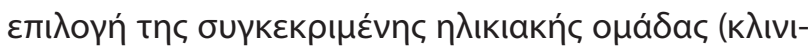

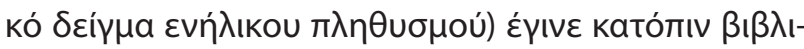

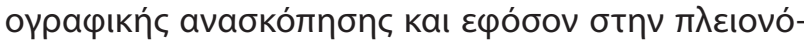

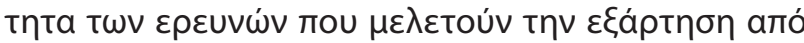

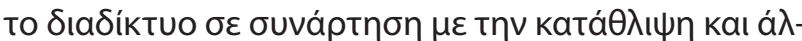

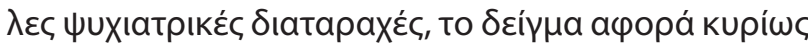

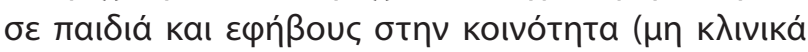

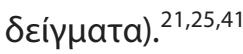

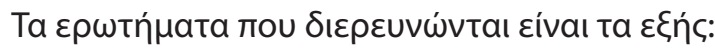

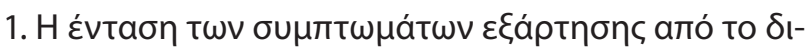

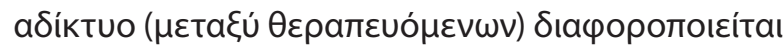

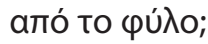

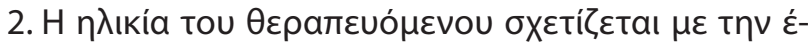

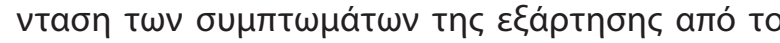

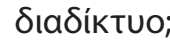

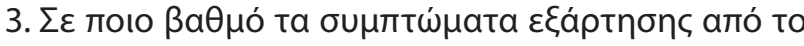

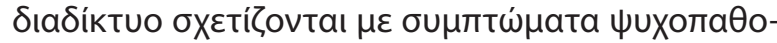

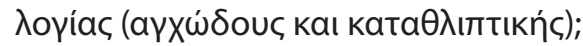

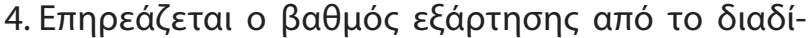

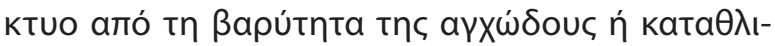

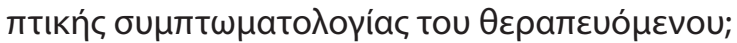

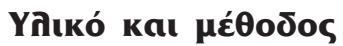

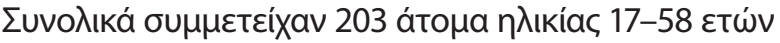

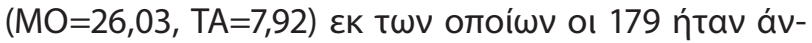

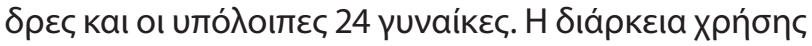

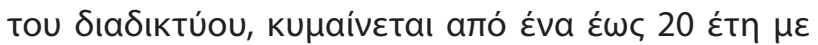

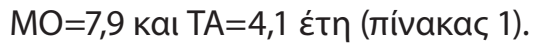

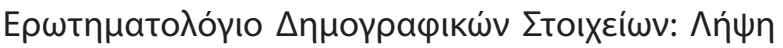

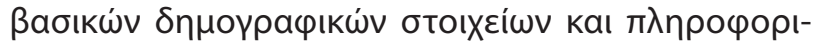

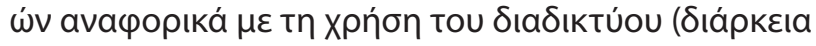

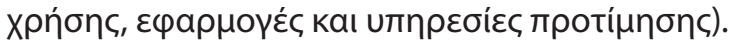

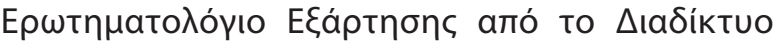

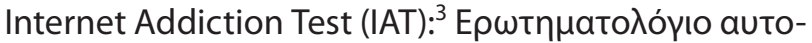

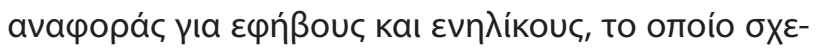

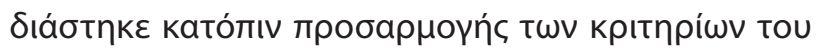

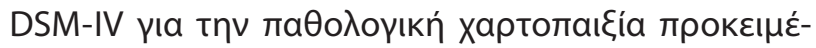

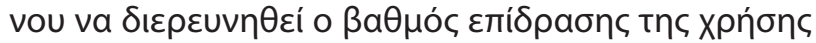

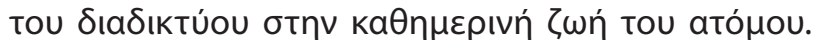

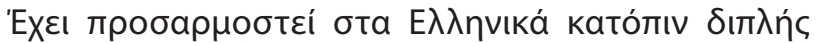

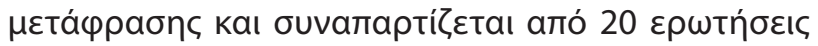

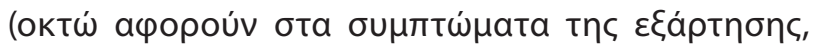

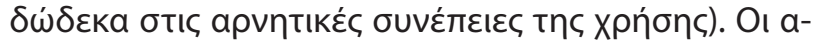

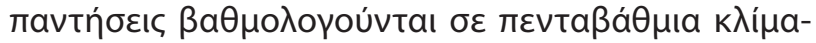

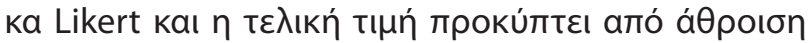

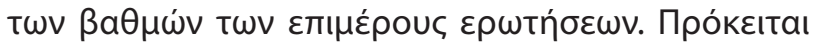

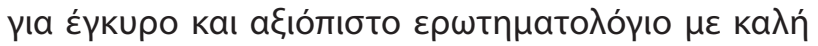

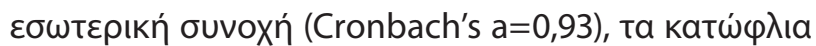

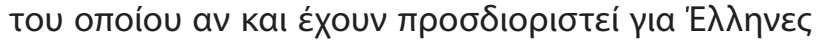

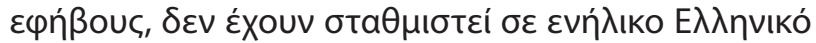
$\pi \lambda \eta \theta u \sigma \mu$. $^{42-43}$

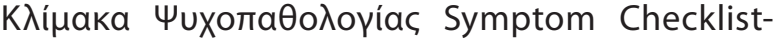

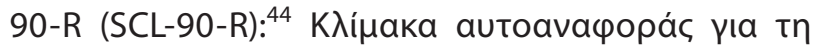

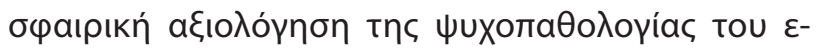

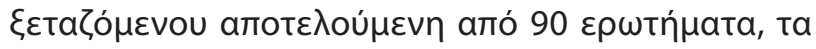

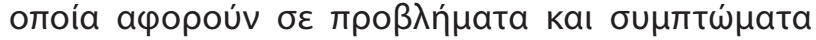

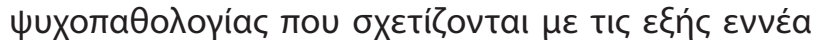

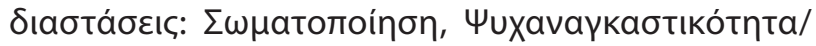

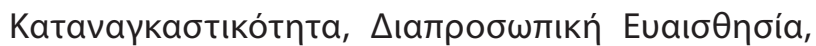

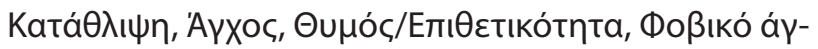

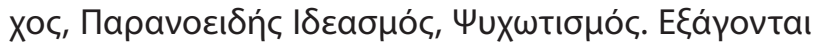

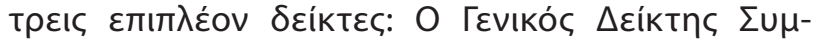

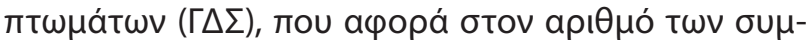

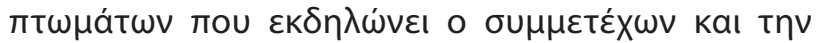




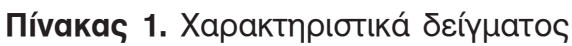

\begin{tabular}{|c|c|c|c|c|c|}
\hline & & & \multicolumn{2}{|c|}{ Фúরo } & \multirow{2}{*}{ ¿uvoגıкó } \\
\hline & & & Гuvaíka & 'Avסраৎ & \\
\hline \multirow{10}{*}{ 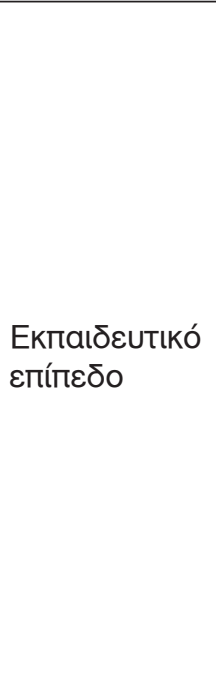 } & \multirow[b]{2}{*}{ 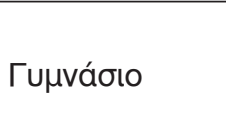 } & $\mathrm{n}$ & 1 & 8 & 9 \\
\hline & & 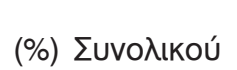 & $0,6 \%$ & $4,7 \%$ & $5,2 \%$ \\
\hline & \multirow{2}{*}{ 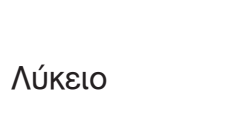 } & $\mathrm{n}$ & 11 & 86 & 97 \\
\hline & & 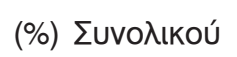 & $6,4 \%$ & $50,0 \%$ & $56,4 \%$ \\
\hline & \multirow{2}{*}{ IEK/Ko入ع́үı૦ } & $\mathrm{n}$ & 5 & 14 & 19 \\
\hline & & 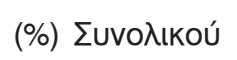 & $2,9 \%$ & $8,1 \%$ & $11,0 \%$ \\
\hline & \multirow[b]{2}{*}{ TEI/AEI } & $\mathrm{n}$ & 3 & 34 & 37 \\
\hline & & 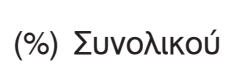 & $1,7 \%$ & $19,8 \%$ & $21,5 \%$ \\
\hline & \multirow[b]{2}{*}{ MeтаптuXıакá } & $\mathrm{n}$ & 1 & 9 & 10 \\
\hline & & 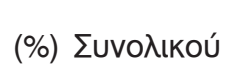 & $0,6 \%$ & $5,2 \%$ & $5,8 \%$ \\
\hline \multirow{2}{*}{\multicolumn{2}{|c|}{ 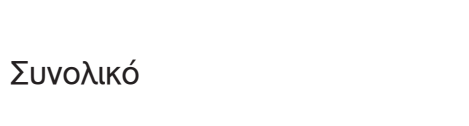 }} & $\mathrm{nN}$ & 21 & 151 & 172 \\
\hline & & 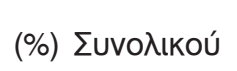 & $12,2 \%$ & $87,8 \%$ & $100,0 \%$ \\
\hline \multicolumn{2}{|l|}{ H入ıкía } & & $\begin{array}{l}\mathrm{MO}=27,9 \\
\mathrm{TA}=10,55\end{array}$ & $\begin{array}{l}\mathrm{MO}=25,77 \\
\mathrm{TA}=7,48\end{array}$ & $\begin{array}{l}\mathrm{MO}=26,02 \\
\mathrm{TA}=7,90\end{array}$ \\
\hline \multicolumn{2}{|c|}{ 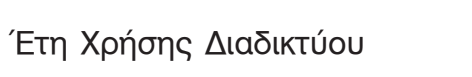 } & & $\begin{array}{l}\mathrm{MO}=5,37 \\
\mathrm{TA}=3,47\end{array}$ & $\begin{array}{l}\mathrm{MO}=8,32 \\
\mathrm{TA}=4,11\end{array}$ & $\begin{array}{l}\mathrm{MO}=7,93 \\
\mathrm{TA}=4,15\end{array}$ \\
\hline
\end{tabular}

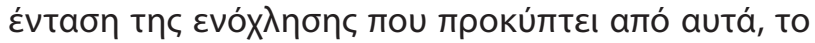

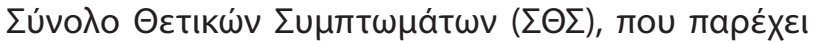

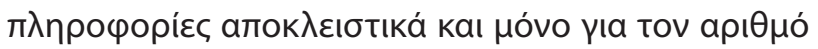

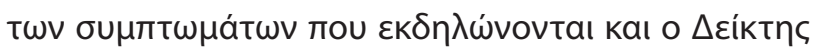

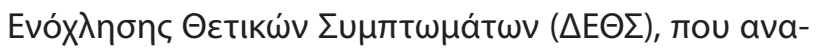

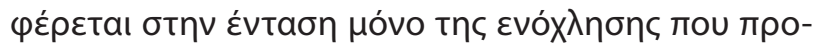

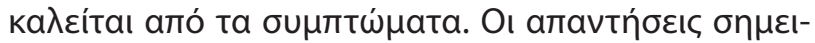

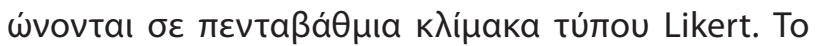

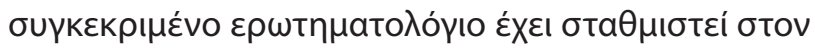

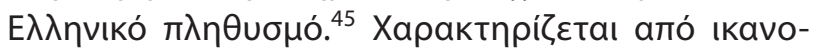

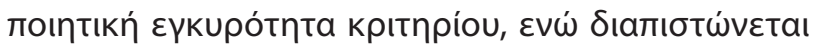

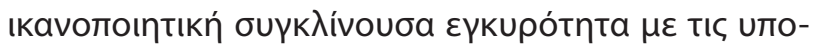

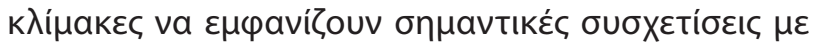

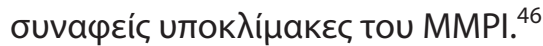

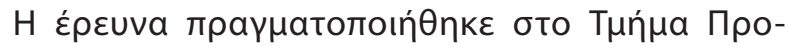

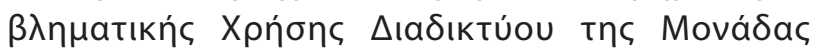

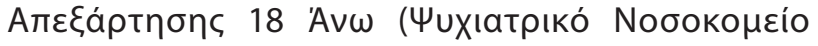

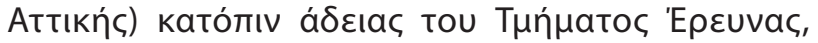

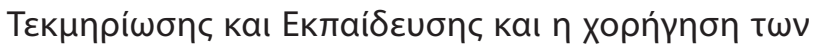

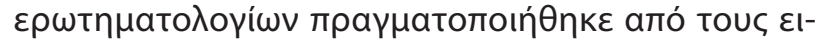

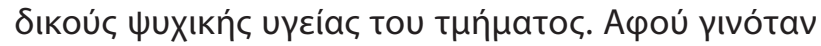

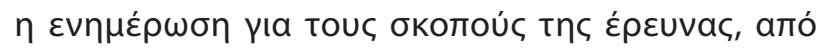

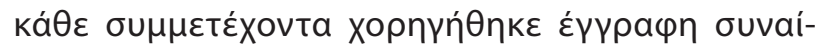

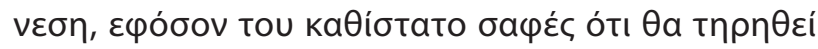

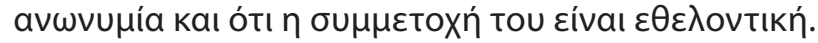

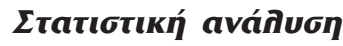

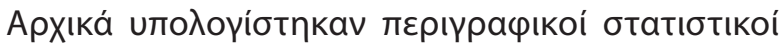

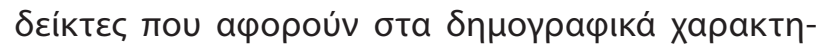

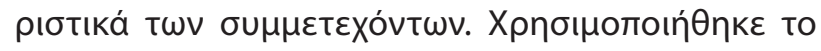

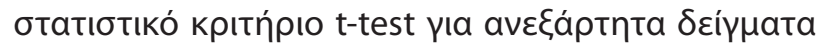

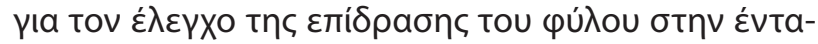

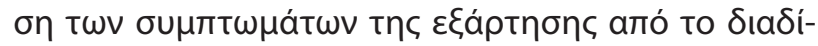

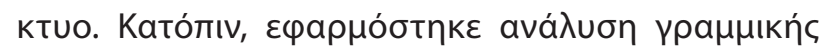

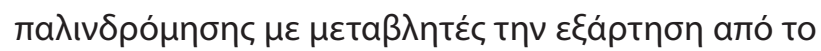

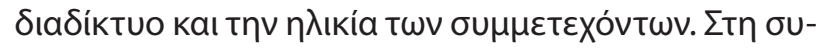

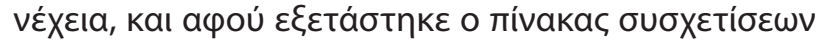

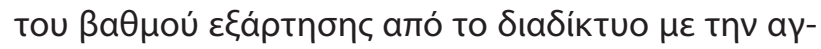




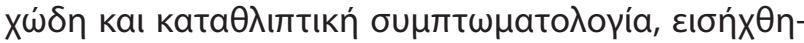

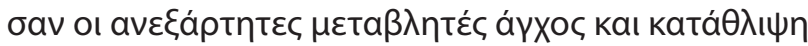

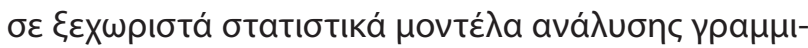

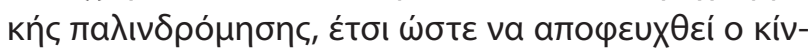

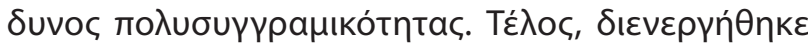

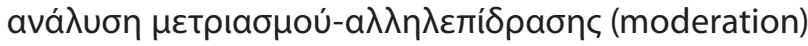

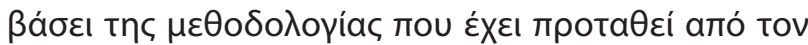

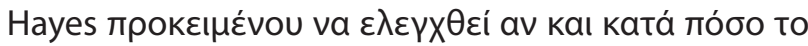

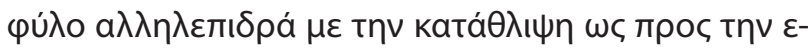

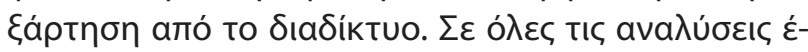

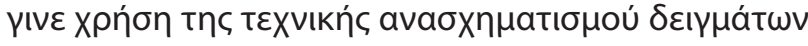

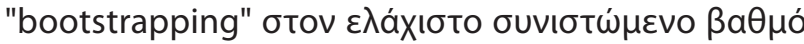

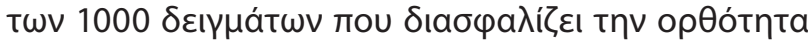

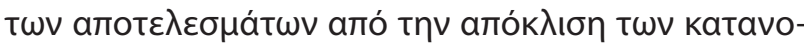

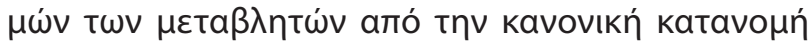

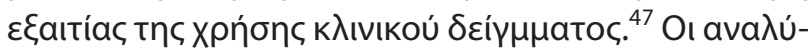

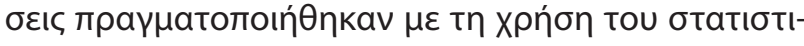

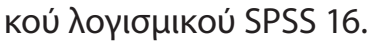

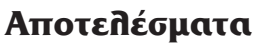

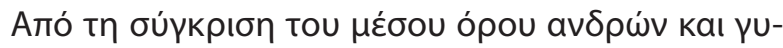

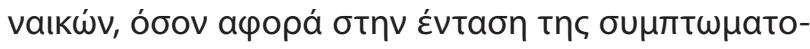

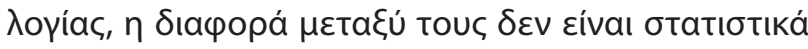

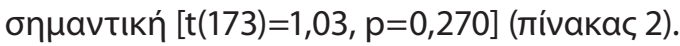

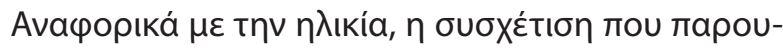

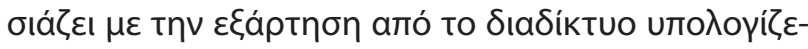

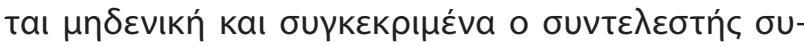

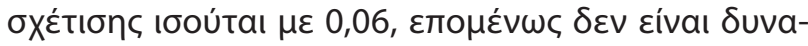

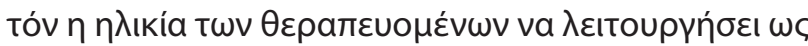

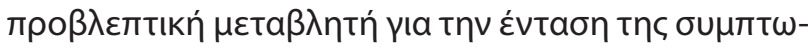

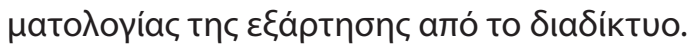

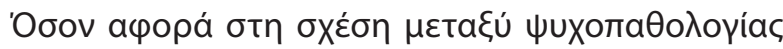

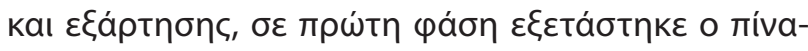

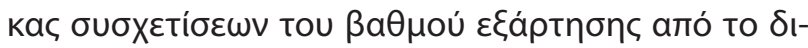

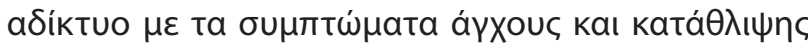
(ті́vakac 3).

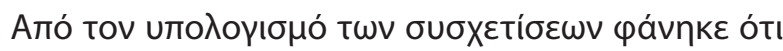

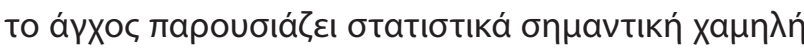

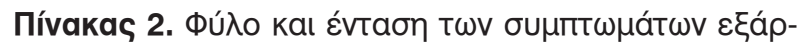

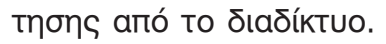

\begin{tabular}{|c|c|c|c|c|}
\hline & Фúภo & $n$ & MO & TA \\
\hline \multirow{2}{*}{ 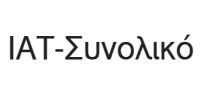 } & Гuvaíka & 19 & 51,1 & 14,7 \\
\hline & 'Avס̄as & 156 & 46,5 & 18,7 \\
\hline
\end{tabular}

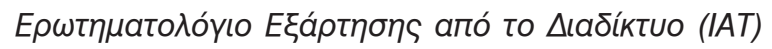

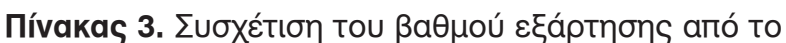

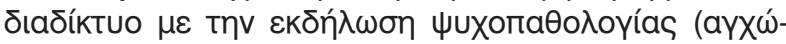

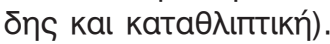

\begin{tabular}{|c|c|c|c|}
\hline & Ката́ $\theta \lambda ı \Psi \eta$ & 'AYXos & IAT-EuvoגıKó \\
\hline 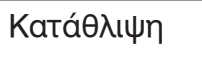 & & 0,07 & 0,12 \\
\hline 'AYXos & & & $0,30^{*}$ \\
\hline 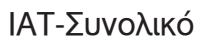 & & & \\
\hline
\end{tabular}

${ }^{*} \mathrm{p}<0,01$

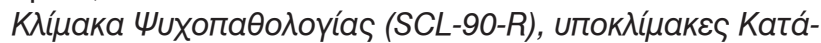
$\theta \lambda ı \psi \eta$ к каı Ayxous

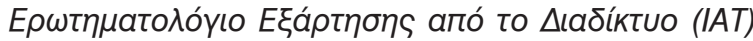

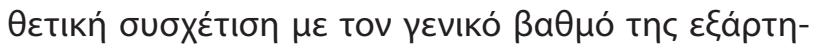

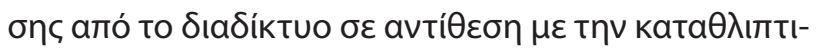

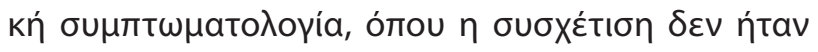

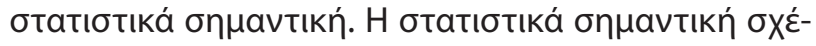

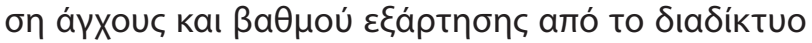

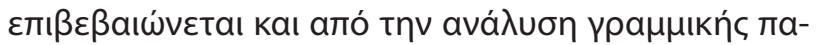

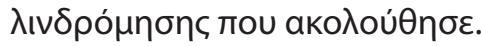

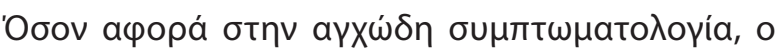

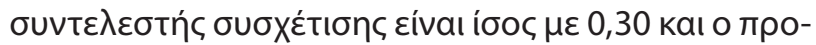

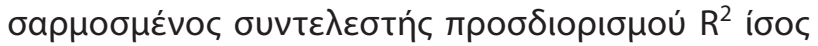

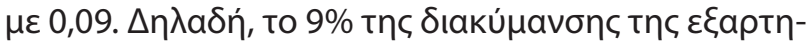

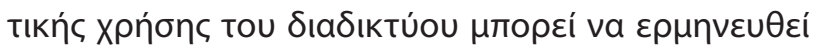

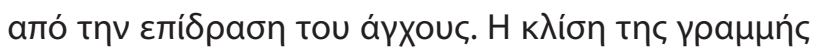

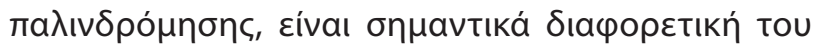

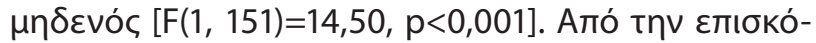

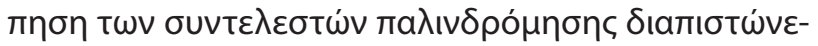
taı ótı тo ápXoৎ ( $\beta=0,30, t=3,81, p<0,01) \sigma \cup \mu \beta a ́ \lambda \lambda \varepsilon ı$

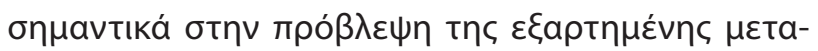

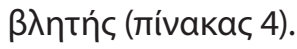

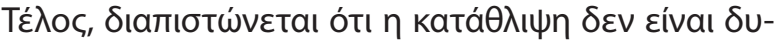

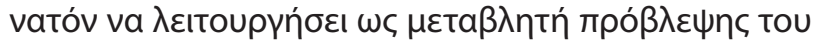

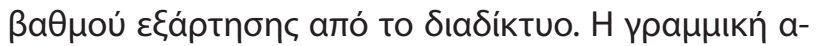

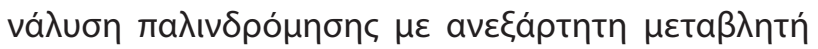

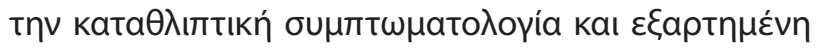

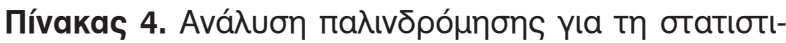

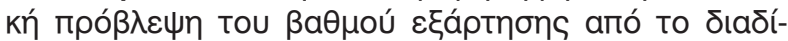

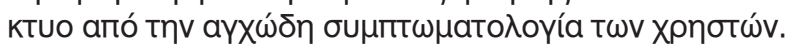

\begin{tabular}{|c|c|c|c|}
\hline 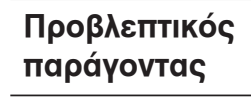 & B & SE B & $\beta$ \\
\hline 'AYXos & 6,70 & 1,76 & 0,30 * \\
\hline
\end{tabular}




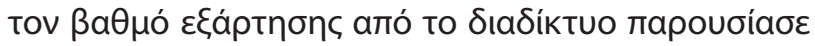

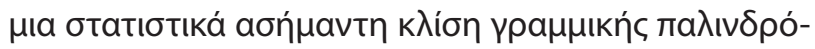

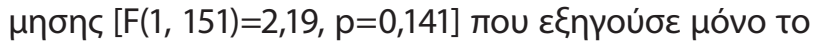

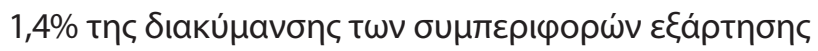

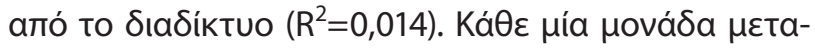

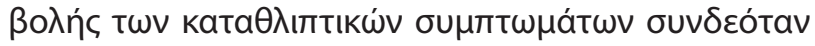

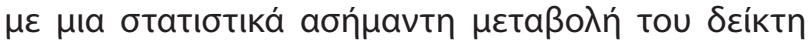

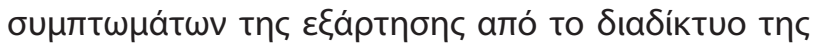

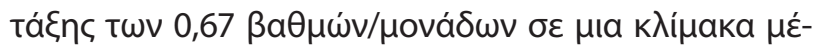

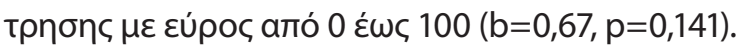

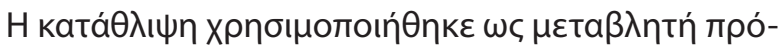

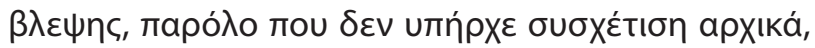

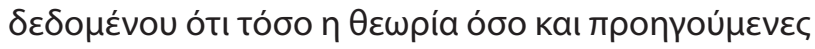

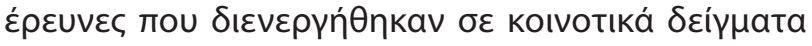

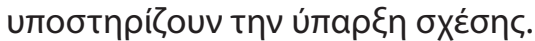

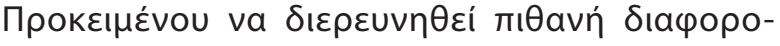

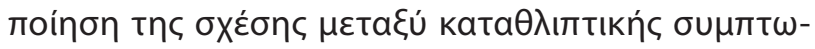

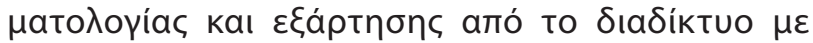

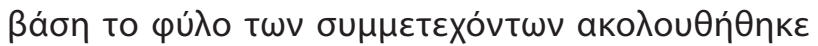

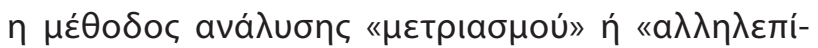

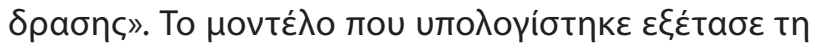

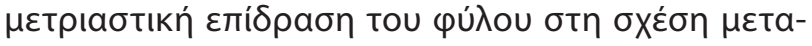

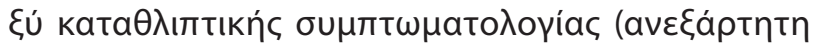

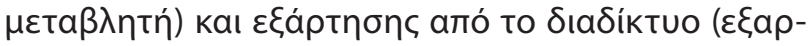

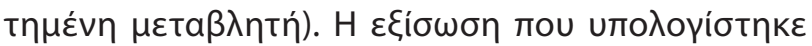

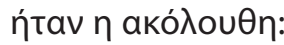

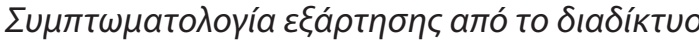

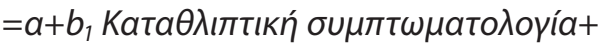

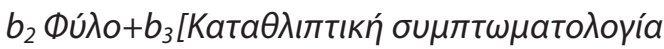

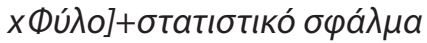

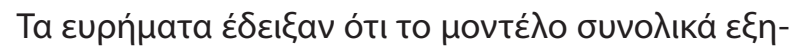

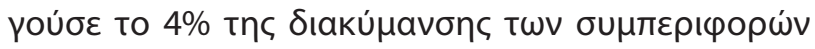

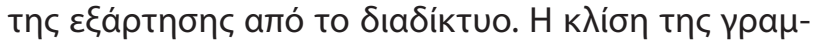

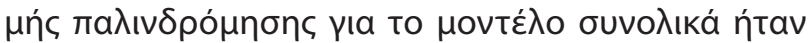

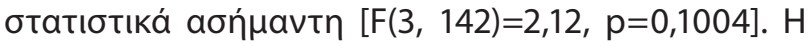

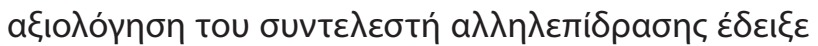

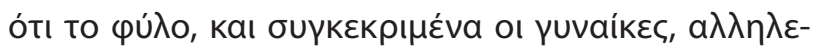

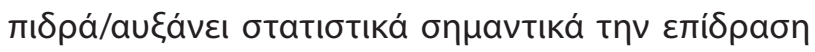

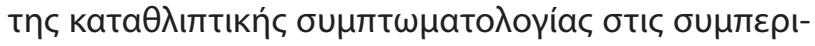

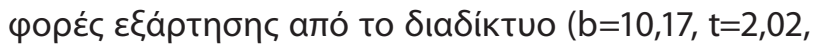

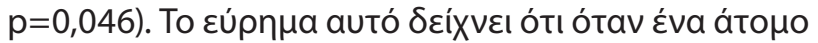

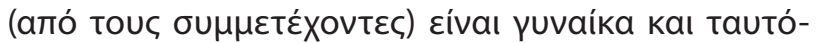

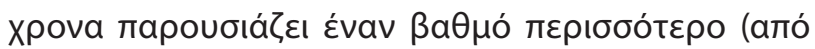

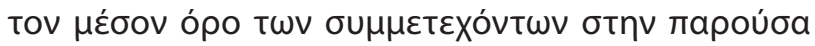

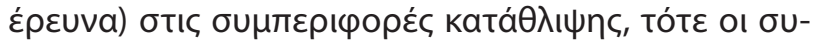

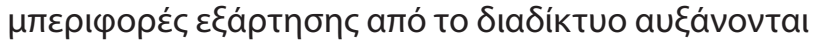

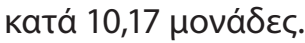

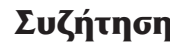

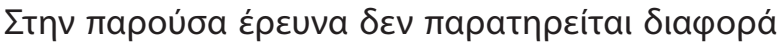

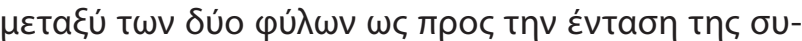

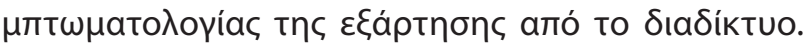

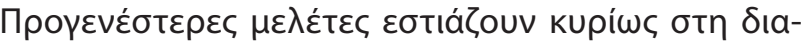

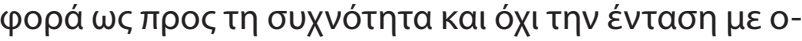

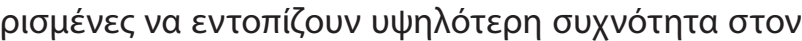

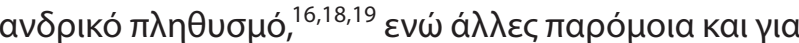

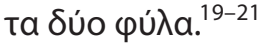

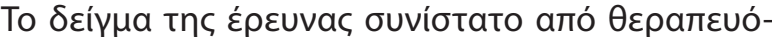

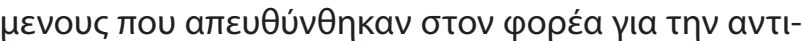

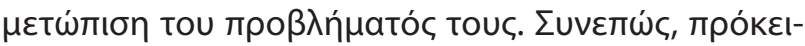

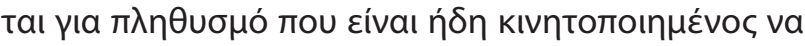

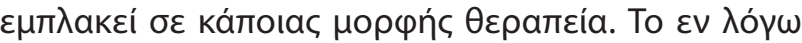

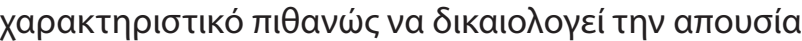

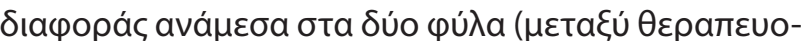

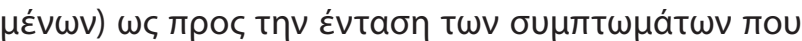

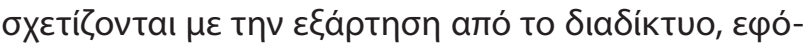

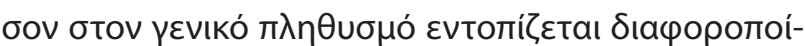

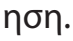

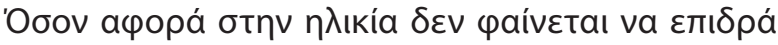

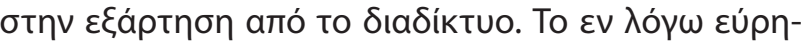

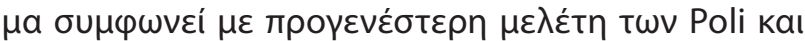

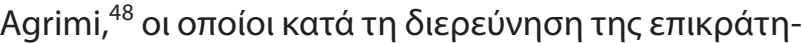

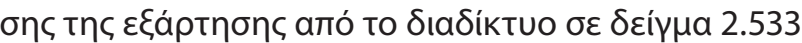

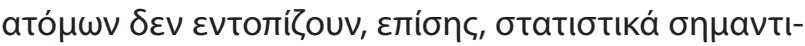

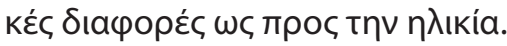

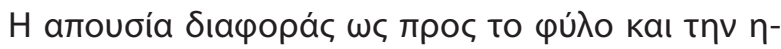

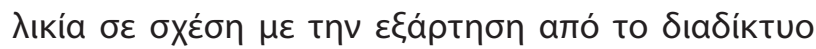

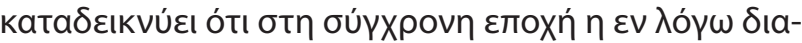

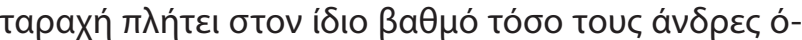

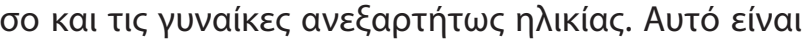

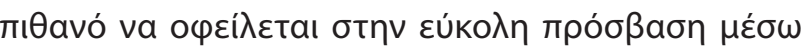

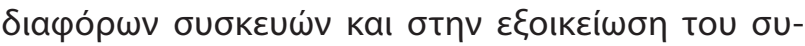

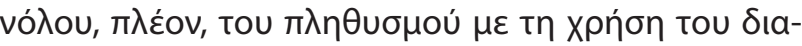

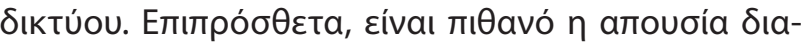

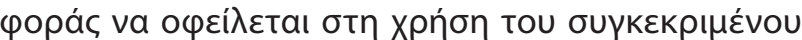

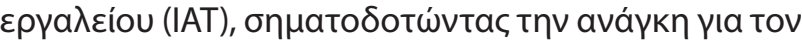

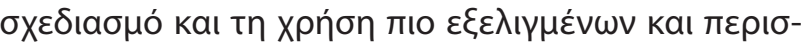

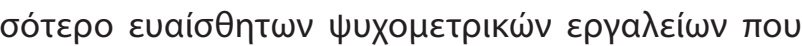

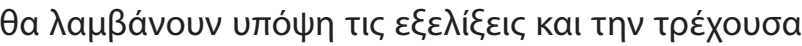
праүнатіко́тпта.

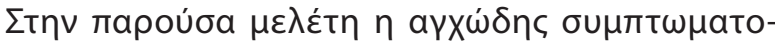

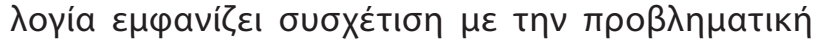

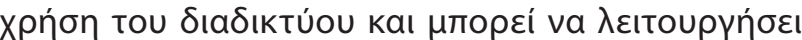

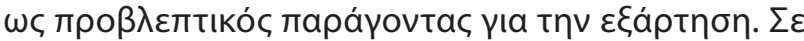




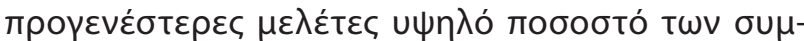

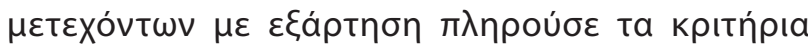

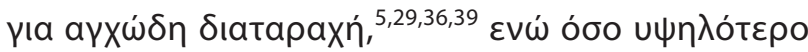

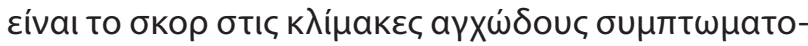

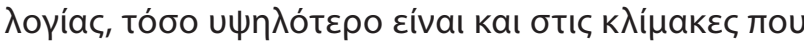

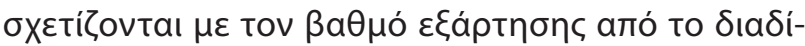
KTUO. ${ }^{36,39}$

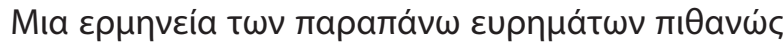

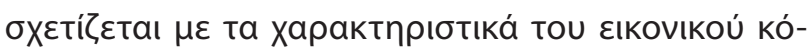

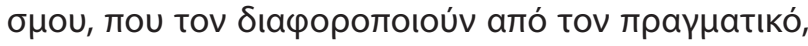

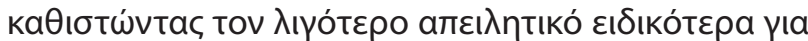

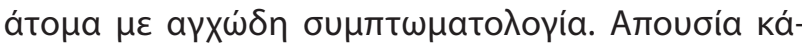

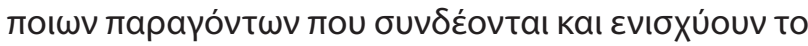

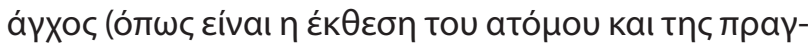

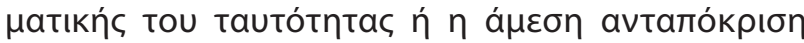

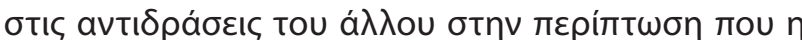

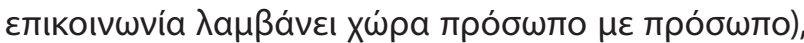

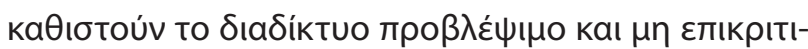

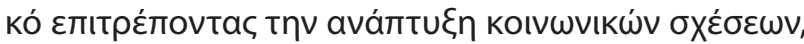

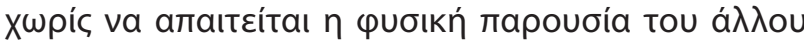

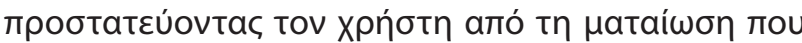

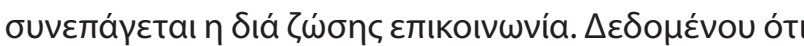

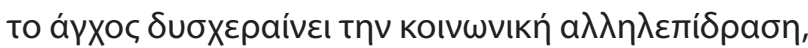

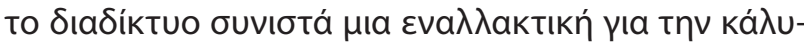

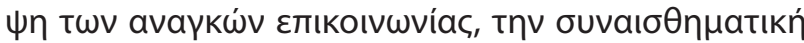

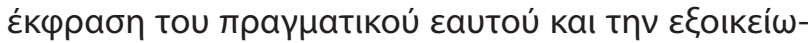

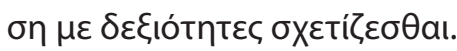

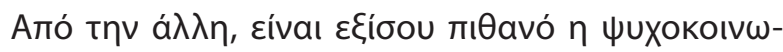

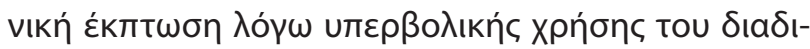

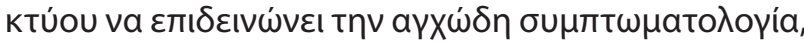

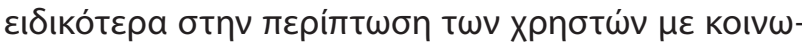

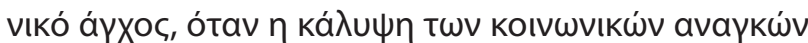

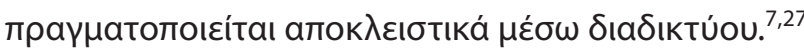

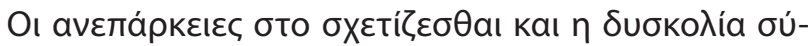

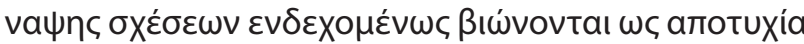

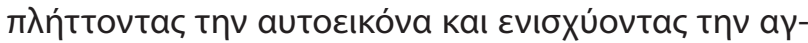

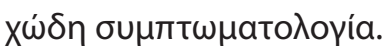

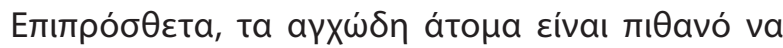

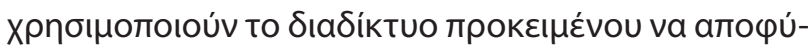

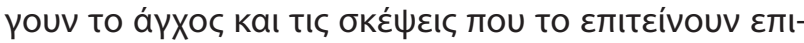

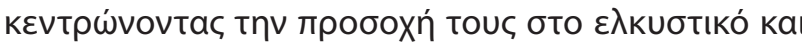

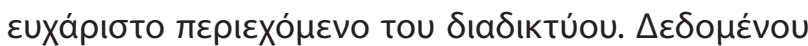

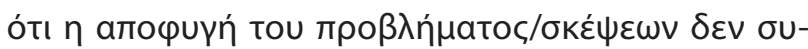

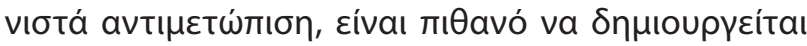

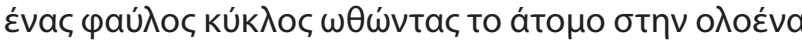

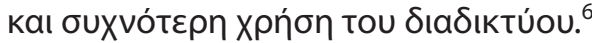

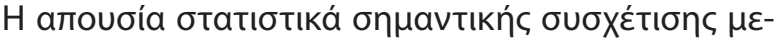

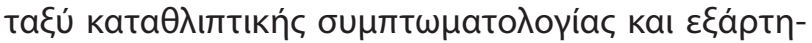

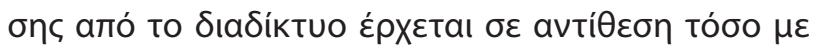

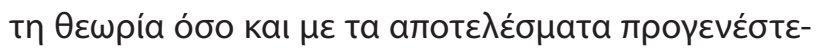

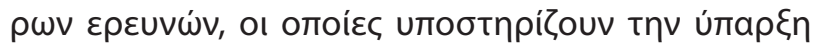

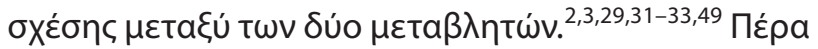

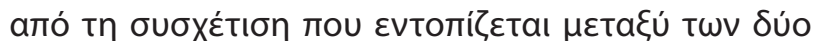

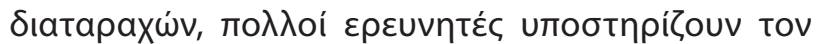

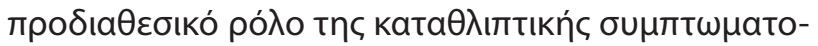

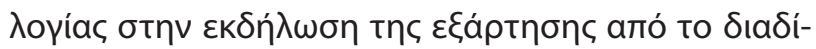

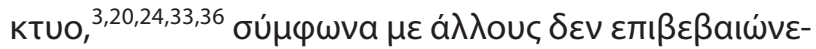

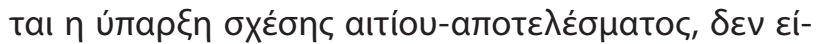

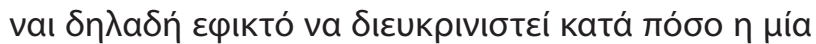

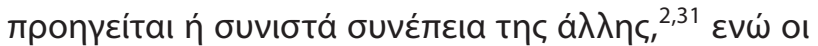

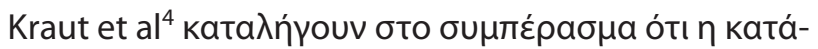

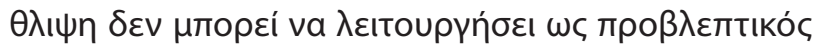

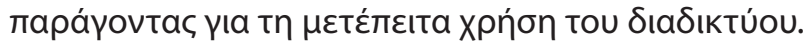

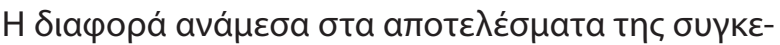

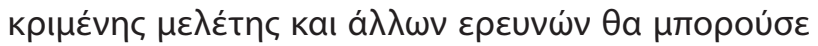

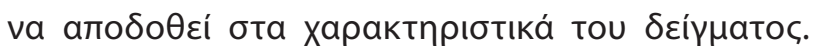

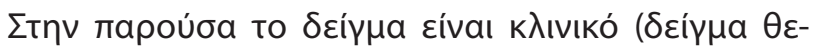

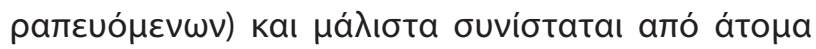

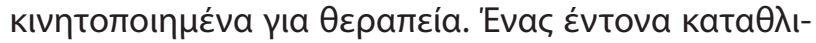

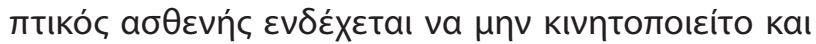

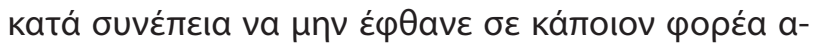

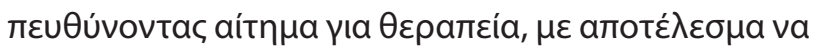

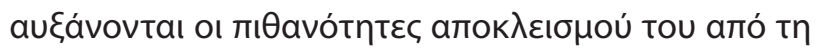

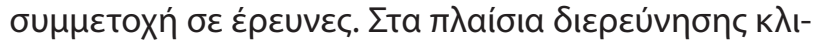

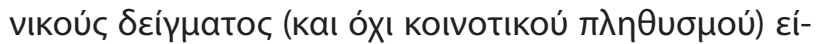

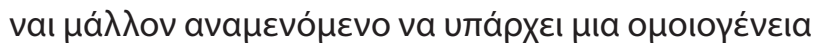

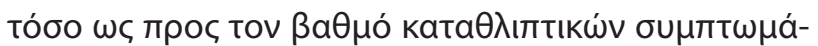

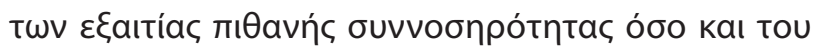

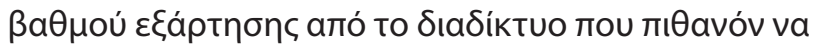

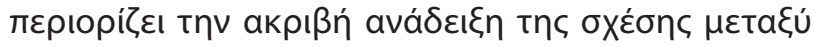

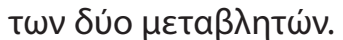

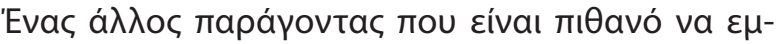

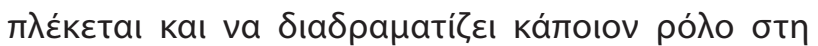

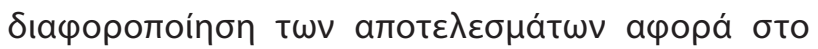

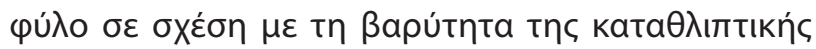

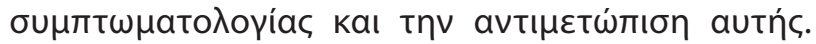

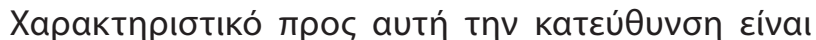

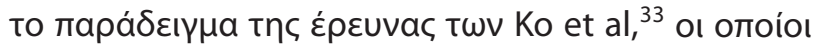

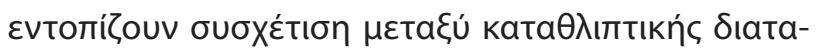

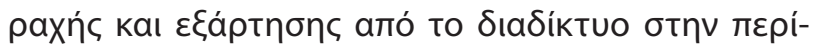

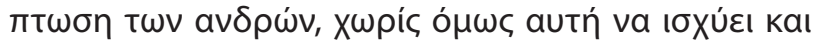

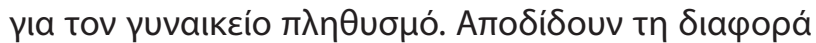

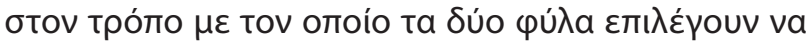




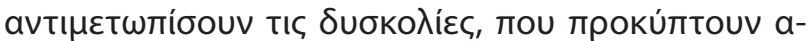

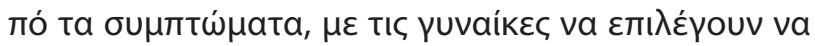

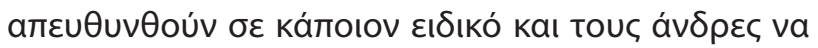

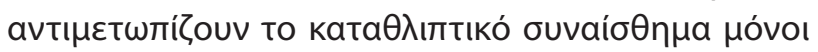

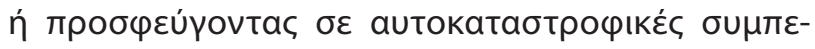

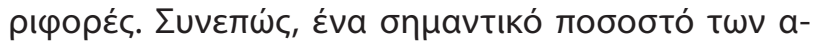

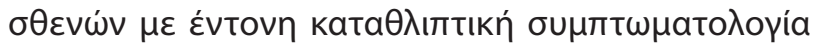

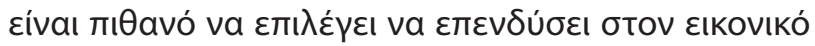

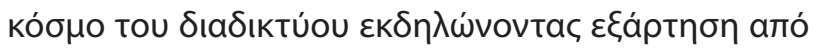

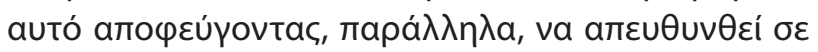

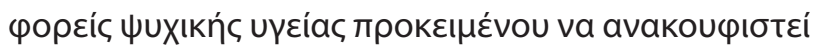
aтó to $\delta$

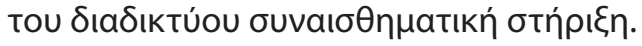

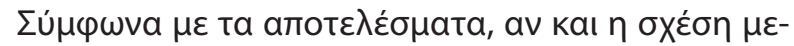

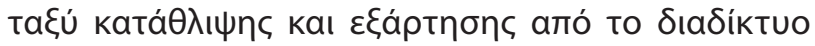

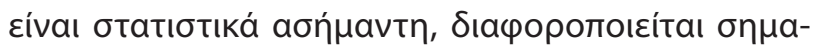

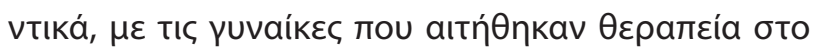

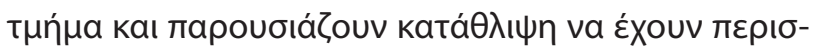

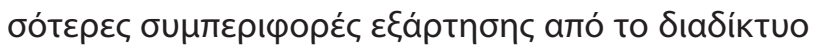

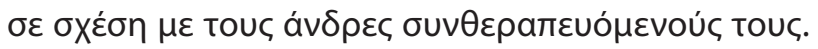

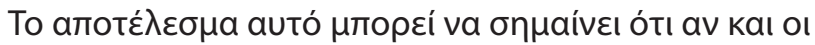

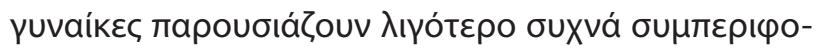

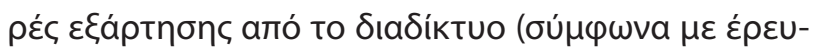

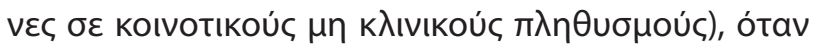

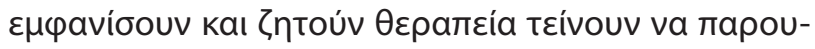

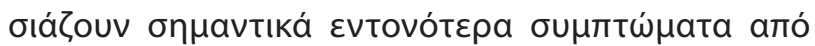

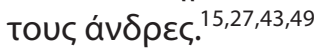

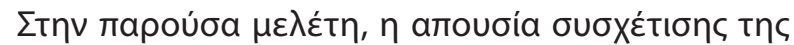
катаӨ

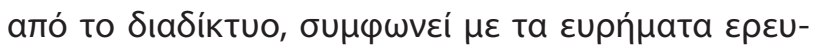

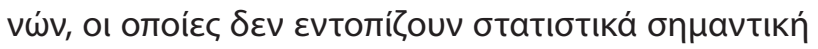

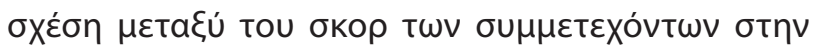

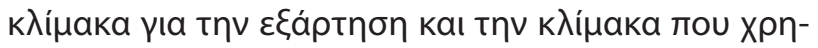

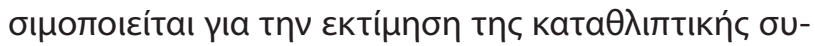

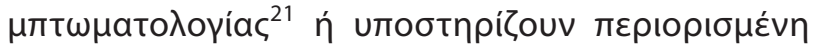

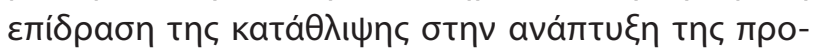

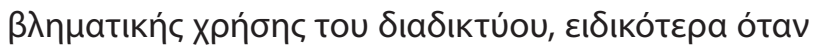

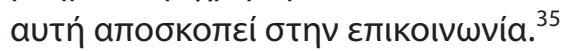

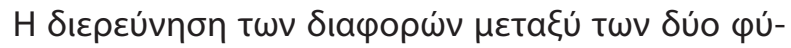

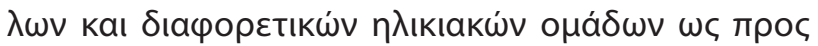

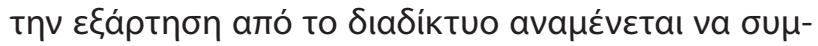

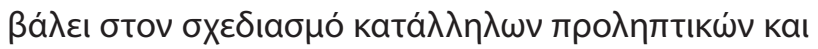

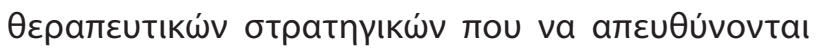

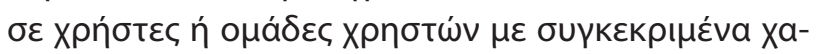

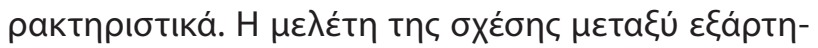

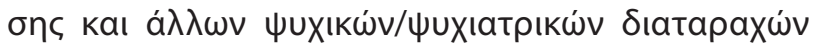

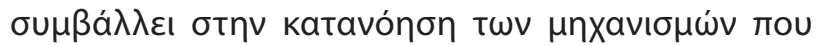

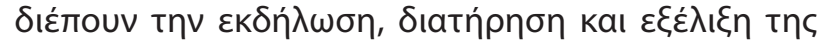

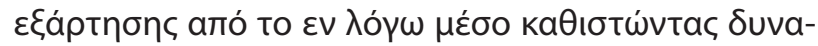

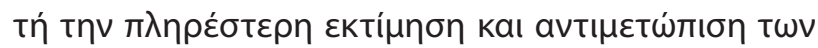

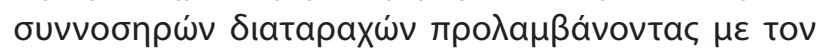

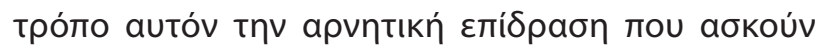

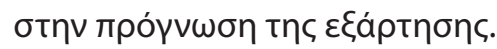

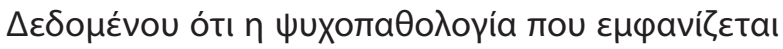

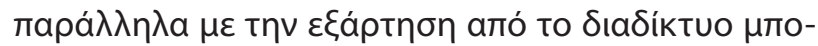

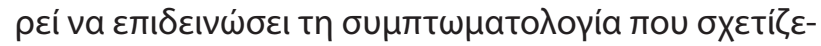

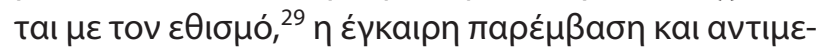

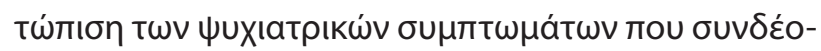

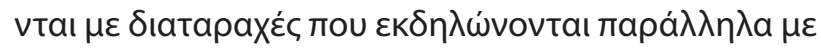

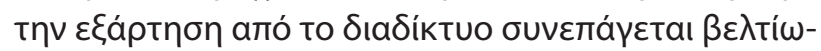

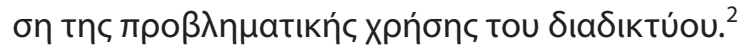

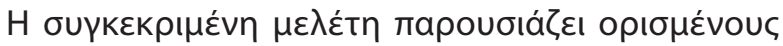

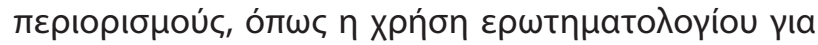

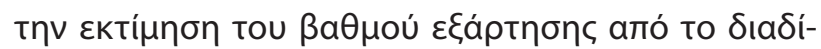

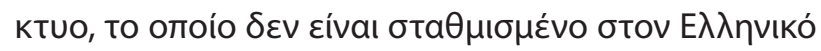

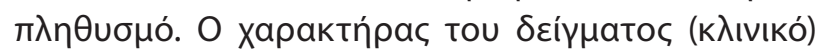

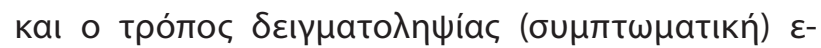

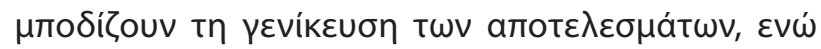

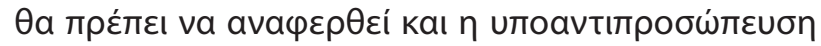

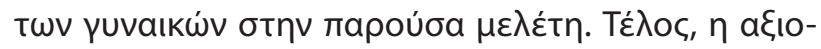

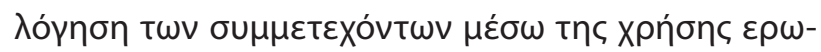

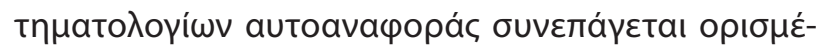

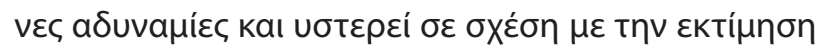

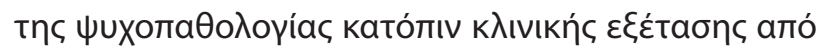

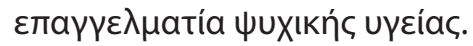

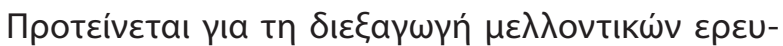

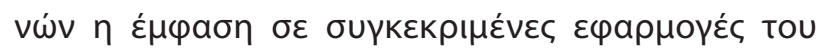

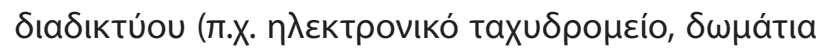

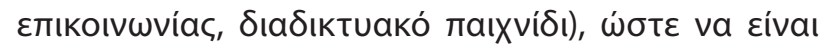

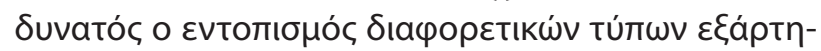

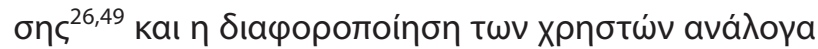

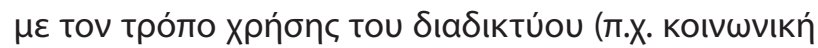

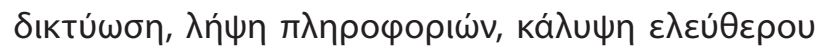

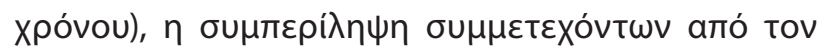

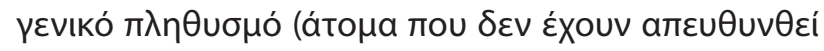

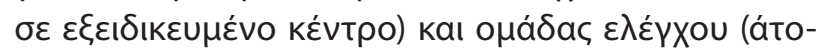

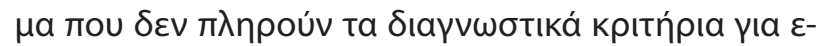

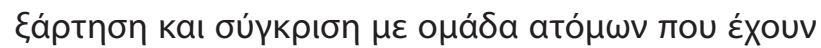

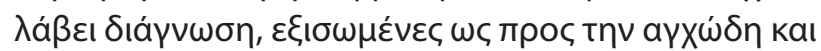

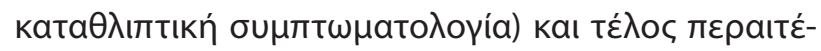

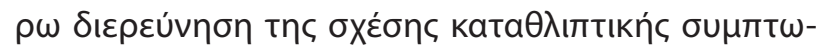

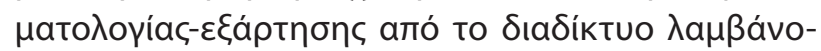

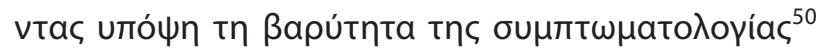

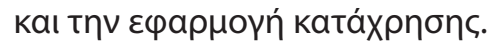




\title{
The relationship of internet addiction with anxiety and depressive symptomatology
}

\author{
E. Soulioti, V. Stavropoulos, S. Christidi, \\ Y. Papastefanou, P. Roussos \\ Department of 18 ANO Dependence Treatment Unit, Department for Problematic Use of Internet (Adults), \\ Psychiatric Hospital of Attica, Athens, Greece
}

Psychiatriki 2018, 29:160-171

\begin{abstract}
Internet stimulates the user's senses causing a variety of subjective experiences and sensations, even though it has no inherent addictive quality. These experiences could be positive, as the improvement of education, or adverse, as the development of internet addiction. There are many people who prefer to invest their time and energy in the virtual world of the internet. They choose to withdraw their emotional investments from face to face communication, while in some cases the internet connection signifies the user's disconnection from the real life, as the person is isolated from the surrounding and lives in a virtual environment. Under these conditions the excessive use of internet may lead to addiction. The purpose of the present study was to investigate the relationship between internet addiction and anxiety and depressive symptomatology of the user. Participants were 203 internet users aged between 17 and 58 years (Mean=26.03, SD=7.92) who approached the Department For Problematic Use Of Internet, Addiction Unit "18ANO" in Psychiatric Hospital Of Attica to receive specialized help for their pathological internet use. Internet Addiction Test (IAT) was used for the assessment of internet addiction and Symptom Checklist90-R (SCL-90-R) was administered for the evaluation of anxiety and depressive symptomatology. The analysis of the survey data showed that gender difference is not observed as to the intensity internet dependence. Younger users are more likely to develop addictive behaviour (in relation to internet use). At this point it should be noted that although positive, this association does not present to be statistically significant. Finally, regarding the relationship between psychopathology and internet addiction, anxiety symptomatology, which was moderately correlated with the overall score at IAT, was found to predict in regression analysis the internet addiction. There was no statistically significant association between internet addiction and depressive symptomatology, with women however, who presented with depressive symptoms to appear more vulnerable than men (who requested therapy from the department). Exploration of the effects of sex and age on internet addiction is expected contribute to the design of the appropriate preventive and therapeutic programs, whereas the study of the relationship between internet addiction and other psychiatric disorders would contribute the understanding of the mechanisms underpinning the development and onset of the addiction.
\end{abstract}

Key words: Internet addiction, psychopathology, anxiety symptoms, depressive symptoms.

\section{BıBntoypacpía}

1. Young KS. Internet addiction: A new clinical phenomenon and its consequences. Am Behav Scient 2004, 48:402-415, doi: $10.1177 / 0002764204270278$

2. Young KS, Rogers RC. The relationship between depression and internet addiction. Cyberpsycholo Behav 1998, 1:25-28, doi: 10.1089/cpb.1998.1.25

3. Young KS. Internet addiction: symptoms, evaluation and treatment. In: Vande-Creek L, Jackson TL (ed) Innovations in clinical practice: a source book. Vol.17 Professional Resource Press, Sarasota FL, 1999:19-31

4. Kraut R, Patterson M, Lundmark V, Kiesler S, Mukophadhyay T, Scherlis W. Internet paradox: a social technology that reduces social involvement and psychological well-being? Am Psychol 1998, 53:1017-1031, PMID: 9841579

5. Shapira NA, Goldsmith TD, Keck PE, Khosia UM, McElroy SL. Psychiatric features of individuals with problematic Internet use. J Affect Disord 2000, 57:267-272, PMID: 10708842 
6. Stavropoulos V, Gentile D, Motti-Stefanidi F. A multilevel Iongitudinal study of adolescent internet addiction: The role of obsessive-compulsive symptoms and classroom openness to experience. Eur J Dev Psychol 2016, 13: 99-114, doi: 10.1186/ s12991-017-0165-z

7. Stavropoulos V, Gomez R, Steen E, Beard C, Liew L, Griffiths MD. The longitudinal association between anxiety and Internet addiction in adolescence: The moderating effect of classroom extraversion. J Behav Addict 2017, 6:237-247, doi: 10.1556/2006.6.2017.026

8. Kuss DJ, Griffiths MD, Pontes HM. Chaos and confusion in DSM-5 diagnosis of Internet Gaming Disorder: Issues, concerns, and recommendations for clarity in the field. J Behav Addict 2017, 6:103-109, doi: 10.1556/2006.5.2016.062

9. Griffiths MD, Kuss DJ, Billieux J, Pontes HM. The evolution of Internet addiction: A global perspective. Addict Behav 2016, 53: 193-195, doi: 10.1016/j.addbeh.2015.11.001

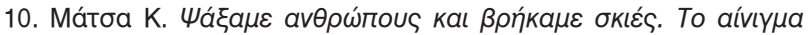

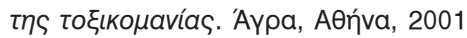

11. Pontes HM, Griffiths MD. Internet addiction disorder and internet gaming disorder are not the same. J Addict Res Ther 2014, 5, doi: 10.4172/2155-6105.1000e124

12. American Psychiatric Association. Diagnostic and Statistical Manual of Mental Disorders. 5th ed. American Psychiatric Publishing, Arlington VA, 2013

13. Christakis DA. Internet addiction: a 21st century epidemic? BMC Medicine 2010, 8:1-3, doi: 10.1186/1741-7015-8-61

14. Ko CH, Yen JY, Yen CF, Chen CS, Che CC. The association between Internet addiction and psychiatric disorder: A review of the literature. Eur Psychiatry 2012, 27:1-8, doi: 10.1016/j. eurpsy.2010.04.011

15. Siomos D, Dafouli E, Braimiotis D, Mouzas O, Angelopoulos N. Internet addiction among Greek adolescent students. Cyberpsych Behav 2008, 11:653-657, Available from: http:// www.academia.edu/4032228/Internet_addiction_as_an_important_predictor_in_early_detection_of_adolescent_drug_use_ experience-implications_for_research_and_practice

16. Frangos CC, Frangos CC, Kiohos AP. Internet addiction among Greek university students: Demographic Associations with the Phenomenon, using the Greek version of Young's Internet Addiction Scale. Int J Econ Sc App Res 2010, 3:1-35, Available from: http://discovery.ucl.ac.uk/id/eprint/20369

17. Tsitsika A, Janikian M, Schoenmakers TM, Tzavela EC, Olafsson $\mathrm{K}$, Wojcik $\mathrm{S}$ et al. Internet addictive behaviour in adolescence: A cross-sectional study in seven European countries. Cyberpsychol Behav Soc Netw 2014, 17:528-53, doi: 10.1089/ cyber.2013.0382

18. Morahan-Martin J. Caught in the web: Research and criticism of the internet abuse with application to college students. In: Wolfe CR (ed) Learning and teaching on the World Wide Web. Academic Press, United States of America, 2001:191-220, doi: 10.1016/B978-012761891-3/50013-8

19. Yang SC, Tung CJ. Comparison of Internet addicts and nonaddicts in Taiwanese high school. Comput Hum Behav 2007, 23:79-96, Available from: https://pdfs.semanticscholar.org/735 2/925e375549e4a001990ff93af72b7805f149.pdf
20. Andreou E, Svoli H. The association between Internet user characteristics and dimensions of Internet addiction among Greek adolescents. Int J Ment Health Addiction 2013, 11:139-148, doi: 10.1007/s11469-012-9404-3

21. Kim K, Ryu E, Chon MY, Yeun EJ, Choi SY, Seo JS. Internet addiction in Korean adolescents and its relation to depression and suicidal ideation: A questionnaire survey. Int $J$ Nurs Stud 2006, 42:185-192

22. Koukia E, Mangoulia P, Alexiou E. Internet addiction and psychopathological symptoms in Greek university students. J Addict Behav Ther Rahabil 2014, 3:1-5, doi: 10.4172/23249005.1000125

23. Canan F, Ataoglu A, Ozcetin A, Icmeli C. The association between internet addiction and dissociation among Turkish college students. Compr Psychiatry 2012, 53:422-426, doi: 10.1016/ j.comppsych.2011.08.006

24. Evren C, Dalbudak E, Evren B, Demirci AC. High risk of Internet addiction and its relationship with lifetime substance use, psychological an behavioral problems among 10th grade adolescents. Psychiat Danub 2014, 26:330-339, Available from: file:///C:/Users/HPS-Journal/Downloads/Dnb_26_4_330_9 Evr\%20(2).pdf

25. Yates TM, Gregor MA, Haviland MG. Child maltreatment, alexithymia and problematic Internet use in young adulthood. Cyberpsych Beh Soc N 2012, 15:219-225, doi: 10.1089/cyber. 2011.0427

26. Widyanto L, Griffiths MD. Internet addiction: a critical review. Int J Ment Health Ad 2006, 4:31-51, Available from: http://www. academia.edu/429533/Widyanto_L._and_Griffiths_M.D._2006_. Internet_addiction_A_critical_review._International_Journal_of_ Mental_Health_and_Addiction_4_31-51

27. Anderson EL, Steen E, Stavropoulos V. Internet use and problematic internet use: A systematic review of longitudinal research trends in adolescence and emergent adulthood. Intern J Adolesc Youth 2016, 1-25, doi: 10.1080/02673843.2016.1227716

28. Block JJ. Issues for DSM-V: Internet addiction. Am J Psychiatry 2008, 165:306-307, doi: 10.1176/appi.ajp.2007.07101556

29. Floros G, Siomos K, Stogiannidou A, Giouzepas I, Garyfallos G. Comorbidity of psychiatric disorders with Internet addiction in a clinical sample: The effect of personality, defense style and psychopathology. Addict Behav 2014, 39:1839-1845, doi: 10.1016/j.addbeh.2014.07.031

30. Bernardi S, Pallanti S. Internet addiction: a descriptive clinical study focusing on comorbidities and dissociative symptoms, Compr Psychiatry 2009, 50:510-516, doi: 10.1016/j. comppsych.2008.11.011

31. Lee YS, Han DH, Yang KC, Daniels MA, Na C, Kee BS Depression like characteristics of 5HTTLPR polymorphism and temperament in excessive internet users. J Affect Disord 2008, 109:165-169, doi: 10.1016/j.jad.2007.10.020

32. Morrison $\mathrm{CM}$, Gore $\mathrm{H}$. The relationship between excessive internet use and depression: A questionnaire-based study of 1319 young people and adults. Psychopathology 2010, 43:121-126, doi: 10.1159/00027700

33. Ko CH, Yen JY, Chen CS, Chen CC, Yen CF. Psychiatric comorbidity of internet addiction in college students: an interview study. CNS Spectr 2008, 13:147-153, PMID: 18227746 
34. Whang LS, Lee S, Chang G. Internet over users' psychological profiles: a behavior sampling analysis on internet addiction. Cyberpsychol Behav 2003, 6:143-150, doi: 10.1089/109493103321640338

35. Caplan SE. Preference for online social interaction: a theory of problematic internet use and psychosocial well-being. Comm Res 2003, 30:625-648, Available from: https://soic.iupui.edu/ files/Thesis_content_example2.pdf

36. Akin A, Iskender M. Internet addiction and depression, anxiety and stress. IOJES 2011, 3:138-148, Available from: http://citeseerx. ist.psu.edu/viewdoc/download?doi=10.1.1.658.6294\&rep=rep1\&t ype $=$ pdf

37. McKenna KYA, Bargh JA. Plan 9 from cyberspace: The implications of the Internet for personality and social psychology. PSPR 2000, 4:57-75, doi: 10.1207/S15327957 PSPR0401_6

38. Kraut R, Kiesler S, Boneva B, Cummings J, Helgeson V, Crawford A. Internet paradox revisited. J Soc Issues 2002, 58: 49-74, doi: 10.1111/1540-4560.00248

39. Hardie E, Tee MY. Excessive Internet Use: The role of personality, Ioneliness and social support networks in Internet addiction. AJETS 2007, 5:34-47, Available from: http://ictaugustine. pbworks. com/f/Excessive+Internet+Use.pdf

40. Montag C, Jurkiewicz M, Reuter M. Low self-directedness is a better predictor for problematic internet use than high neuroticism. Comput Human Behav 2010, 26:1531-1535, doi: 10.1016/j. chb.2010.05.021

41. Siomos K, Floros G, Fisoun V, Dafouli E, Farkonas N, Sergentani $\mathrm{E}$ et al. Evolution of internet addiction in Greek adolescent students over a two-year period: the impact of parental bonding. Eur Child Adolesc Psychiatry 2012, 21:211-219, Available from: https://www.researchgate.net/publication/221811535_Evolution_ of_Internet_addiction_in_Greek_adolescent_students_over_a_ two-year_period_The_impact_of_parental_bonding

42. Leung L, Lee PSN. Impact of internet literacy, internet addiction symptoms, and internet activities on academic performance. Soc Sci Comput Rev 2012, 30:403-418, doi: 10.1177/ 0894439311435217

43. Stavropoulos V, Alexandraki K, Motti-Stefanidi F. Recognizing internet addiction: Prevalence and relationship to academic achievement in adolescents enrolled in urban and rural Greek high schools. J Adolesc 2013, 36:565-576

44. Derogatis LR. Symptom Checklist-90-R: Administration, scoring and procedures manual. 3rd ed. National Computer Systems Inc, Minneapolis, 1994

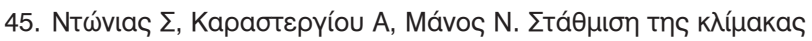

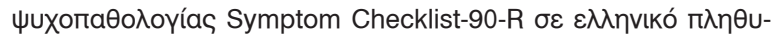

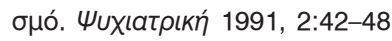

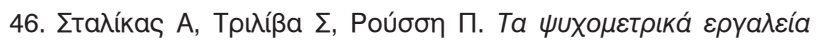

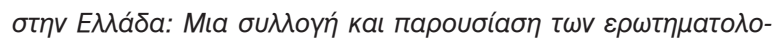

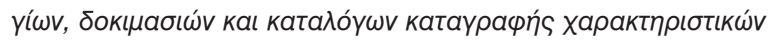

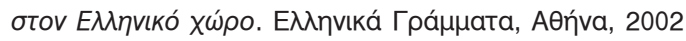

47. Berkovits I, Hancock GR, Nevitt J. Bootstrap resampling approaches for repeated measure designs: Relative robustness to sphericity and normality violations. Educ Psychol Meas 2000, 60:877-892, doi: 10.1177/00131640021970961

48. Poli R, Agrimi E. Internet addiction disorder: Prevalence in an Italian student population. Nord J Psychiatry 2012, 66: 55-59, doi: 10.3109/08039488.2011.605169

49. Kuss DJ, Griffiths MD, Binder JF. Internet addiction in students: Prevalence and risk factors. Comput Human Behav 2013, 29:959-966, doi: 10.1016/j.chb.2012.12.024

50. Bessière K, Kiesler S, Kraut R, Boneva BS. Effects of Internet use and social resources on changes in depression. Inform Commun \& Soc 2008, 11:47-70, doi: 10.1080/13691180701858851

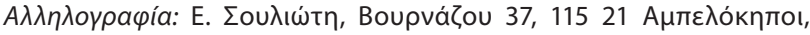

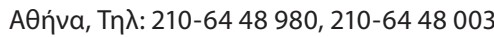

e-mail: net18ano@gmail.com,www.18ano.gr 